\title{
Propagation of weak shocks through a random medium
}

\author{
By LAMBERTUS HESSELINK† \\ AND BRADFORD STURTEVANT \\ Graduate Aeronautical Laboratories, California Institute of Technology, \\ Pasadena, CA 91125, USA
}

(Received 15 April 1985 and in revised form 6 April 1988)

The propagation of weak shock waves $\left(M_{\mathrm{s}}=1.007,1.03\right.$ and 1.1) through a statistically uniform random medium has been investigated experimentally in a shock tube. The wave-front geometry, rise time and amplitude of initially plane shocks which have propagated through a random mixture of helium and refrigerant 12 are measured. The effect of shock propagation on the properties of the random medium is visualized with schlieren and shadow photography. The pressure histories of the distorted shock waves reflecting from a normal end wall are observed to be both peaked and rounded. In the rounded case the perturbed shock is found to be made up of a succession of weak, slightly curved fronts with a total effective rise time orders of magnitude greater than the classical Taylor thickness. The radius of curvature of the weakest shocks after propagating through the random medium is inferred from observations at two downstream stations to be about 7 times the integral scale of the gas inhomogeneities. It is concluded that the observed distortions of the wave fronts can best be explained in terms of random focusing and defocusing of the front by the inhomogeneities in the medium. A ray-tracing calculation has been used to interpret the experimental observations. It is found that geometrical considerations are sufficient to account for many of the effects observed on the shocks.

\section{Introduction}

The propagation of a shock wave through a random medium is an example of a nonlinear interaction process in which a pressure field is coupled to vorticity and/or entropy fluctuations. The shock wave has an effect on the fluctuations in the fluid and, conversely, fluid non-uniformities affect the shock propagation. Both effects are particularly important in flows in which the shocks are of moderate strength (not so strong that the stability of shock waves predominates) and the non-uniformities are large. In this situation the basic nonlinearity of fluid mechanics is important both in the wave propagation and in the motion of the turbulent fluid. On the other hand, the interaction of acoustic waves with non-uniform media can be treated by (linear) scattering theory and the wave propagation has no effect on the non-uniformities. For moderate-strength shocks departures from the predictions of acoustic theory are due to the effects of nonlinear wave steepening, and the wave can substantially enhance turbulent mixing.

There is an increasing number of applications in which incomplete understanding

$\uparrow$ Present address : Departments of Aeronautics and Astronautics, and of Electrical Engineering, Stanford University, Stanford, CA 94305-4035, USA 
of the coupling between finite-amplitude waves and fluid-dynamic non-uniformity is a limiting factor, including high-energy flowing-gas lasers, laser fusion, combustion instability, shock/boundary-layer interaction, the propagation of the sonic boom through the Earth's atmosphere, supersonic jet noise, and even supernovae explosions. In this paper we describe an experimental investigation of the propagation of weak shocks through a statistically uniform laboratory-generated random medium. The experiments are designed to investigate some of the general features of the interaction phenomena.

Aspects of the physical processes involved in the interaction between shock waves and turbulence have been studied previously both theoretically and experimentally. Chu \& Kovasznay (1957) established a convenient basis for cataloguing wave interactions and showed how, to second order, nonlinear interactions in a viscous compressible fluid act like 'source' terms in the mass, momentum and energy equations. Chu \& Kovasznay split the flow field into three contributions or 'modes': the vorticity mode, the sound or wave mode and the entropy mode; and they studied the effects of the interaction of these modes. They showed that the vorticity mode can be altered by vortex stretching, by nonlinear interactions between waves (e.g. Mach reflection), and by wave-entropy interactions through the Bjerknes term in the vorticity equation. The wave mode can be modified by nonlinear wave interactions (parametric amplification), by scattering from vorticity and entropy fluctuations and by the generation of aerodynamic noise by Reynolds stresses in the vortical velocity field. Finally, modifications of the entropy field are caused by convection induced by the waves and by vorticity through the process of turbulent heat transfer and mixing.

Wave-mode interactions have been studied by Lighthill (1953) and Ribner (1954). In these works the emphasis was on the calculation of the scattered wave field. Crow (1969) reinterpreted the Lighthill theory and applied it to the sonic-boom problem. Single-scattering theory explains many of the observed features in sonic-boom pressure profiles (Maglieri \& Parrot 1963), but predicts impossibly large perturbations on the shock front. Furthermore, in some cases documented by Maglieri \& Parrot, the rise time of the initial disturbance on the sonic boom $\mathrm{N}$-wave is observed to be up to three orders of magnitude greater than the Taylor shock thickness. $\uparrow$ Crow has pointed out that second-order theory contains a mechanism for thickening shocks, and Plotkin \& George (1972) developed a second-order perturbation scheme which led to a Burgers equation for the wave structure, with the molecular viscosity replaced by a viscosity-like term depending on the properties of the Earth's turbulent boundary layer. They calculated the equilibrium wave-front structure (governed by the balance between nonlinear steepening and turbulent scattering of acoustic energy) of the mean coherent wave front, and showed that the profile of an initially discontinuous wave becomes smooth and rather thick. Ffowcs Williams \& Howe (1973) disputed this result and correctly pointed out that an initially discontinuous wave must, in accordance with geometrical acoustics, remain discontinuous. They reinterpreted and reconfirmed this fact using a multiplescattering theory which in the limit is equivalent to linearized geometrical acousties. Using order-of-magnitude estimates they further argued that focusing, a mechanism

$\uparrow$ Hodgson (1973) has suggested that vibrational relaxation effects in oxygen and nitrogen might substantially increase shock thickness. The relaxation frequencies of these gases are strongly dependent upon the water vapour content of air, and it is unlikely that this mechanism can explain the observed thickening in the sonic-boom pressure profiles. 
first proposed by Pierce (1968) to explain the main features of sonic-boom profiles, is unlikely to be important in atmospheric scattering. This paper itself, however, was incorrect, as pointed out by Howe (1973). To explain the observations of the present work we find it necessary to invoke the focusing mechanism.

Concerning the point that linear theory predicts excessive wave-front amplitudes, it should be noted that, even though fluctuations of index of refraction may be small, perturbation theory is not uniformly valid for large path lengths (Keller 1962). Thus, conclusions from linear theory about multiple scattering may be in error, again raising the possibility that nonlinear phenomena such as focusing and caustics are important in the interaction process. Kravtsov (1969) has explicitly considered the possibility that caustics may form in a random medium, but he too uses linearized geometrical acoustics theory. In a paper that specifically addresses the processes thought to be dominant in the present experiments, Kulkarny \& White (1982) showed that, because of the inherent nonlinearity of geometrical acoustics, focusing inevitably occurs along any given ray within a finite distance from the source. They have shown how the mean distance to first focus and the mean density of foci depend on the magnitude and scale of the inhomogeneities in the medium. More recently, White (1987) has expanded this analysis and shown how accurate statistical descriptions of the wave field up to fourth-order moments may be computed using geometrical acoustics, even in the vicinity of caustics.

The effect of wave propagation on fluid motion has also been treated in the literature, including studies of the interaction between shock waves and single scatterers and arrays of discrete inhomogeneities. The influence of the wave mode on the vorticity and entropy modes was studied by Markstein $(1957 a, b)$ and Rudinger (1958). They found that as a consequence of the interaction between a shock wave and a laminar flame front the Rayleigh-Taylor instability (Taylor 1950) $\dagger$ causes the flame front surface area to increase and ultimately results in disintegration of the front. A physical model of the interaction of a shock with a spherical inhomogeneity, in which it was argued that a vortex ring is produced, was put forward by Rudinger \& Sommers (1960). Recently the same problem has been treated experimentally (Haas \& Sturtevant 1987) and by large-scale numerical computation (Picone et al. 1984; Winkler et al. 1987). Wave propagation through an array of vortices was studied by Werner (1961), who computed the transient pressure field but did not treat modifications of the vorticity mode. Dettleff et al. (1979) reported the observation of vortex rings in the fluid behind liquefaction shock waves, presumably caused by some undefined non-uniformity of the preshock fluid. Finally, Anyiwo \& Bushnell (1982) have studied the amplification of turbulence in shock-wave boundary-layer interaction, an important application of the general problem considered here.

In the present investigation we have studied the propagation of relatively weak shock waves through a randomly inhomogeneous mixture of helium (molecular weight 4 ) and refrigerant 12 (molecular weight 121 ). Both the modifications to the pressure field and the density field are studied. For the amplitude of fluid inhomogeneities that can be generated in the present apparatus, namely $6 \%$ fluctuation in the sound speed, only shocks of strength Mach number $M_{\mathrm{s}}<1.1$ are substantially affected by passage through the test region. Stronger shocks are so

\footnotetext{
$\dagger$ Also known as the Richtmeyer-Meshkov instability; of. Richtmyer (1960), Meshkov (1970), Meyer \& Blewett (1972).
} 
stable that scattering is minimal. Thus, acoustic theory, particularly the theories of geometrical acoustics and geometrical diffraction of sound pulses, forms the basis for discussing the experimental configuration and the results of this work. As is usually the case in optics as well as in acoustics, when a sharp discontinuity propagates through a random medium characterized by a spectrum of lengthscales associated with eddies, diffraction effects may be important. The Fraunhofer parameter $F=$ $(A / d) z$, where $A$ represents the thickness of the wave, $d$ is a characteristic lengthscale in the medium and $z$ the propagation distance, is an indicator of the importance of diffraction effects. When $F \gtrsim d$, diffraction effects may be important. In our case $A \approx 0.1 \mathrm{~mm}, d \approx 1 \mathrm{~cm}, z \approx 30 \mathrm{~cm}$, so $F \approx 3 \mathrm{~mm}<d$, which indicates that for these values diffraction is not important. It should be noted, however, that the choice of values for these parameters is not unique, and for other values, e.g., scales $d$ smaller than $3 \mathrm{~mm}$, diffraction needs to be taken into account in order to exactly describe the wave propagation. Furthermore, the geometrical ray-tracing calculations discussed in $\$ 4$ show that wave front focusing occurs within the medium, and near a focus diffraction effects cannot be ignored. However, geometrical arguments yield a qualitative picture of wave-front configurations; as will become apparent below, they are sufficient to describe the salient features of the present observations. This position is supported by recent work of White (1987), who showed that even near a caustic computations of up to the fourth moment may be carried out using geometrical acoustics instead of diffraction analysis. In the present study we have chosen a geometrical acoustics ray-tracing calculation for interpretation of the experimental results. A major result of this study is that most of the observed scattering effects can be interpreted by geometrical considerations, in which the initially discontinuous shocks remain discontinuous but the fronts distort in three dimensions.

\section{Experimental}

\subsection{Description of the facility}

An apparatus in which is generated a standardized model of a random medium was constructed to serve as the test section of the GALCIT 17 inch $(43 \mathrm{~cm})$ diameter shock tube (figure 1) (Hesselink 1977, Appendix A). Its function is to fill a $26.7 \mathrm{~cm}$ square by $35.2 \mathrm{~cm}$ long rectangular volume at atmospheric pressure with two gases incompletely mixed on as small a scale as possible. The apparatus consists of an array of fine jets, alternately of helium and dichlorodifluoromethane $\left(\mathrm{CCl}_{2} \mathrm{~F}_{2}\right.$, hereafter referred to as refrigerant 12 , or R12) arrayed in a checkerboard pattern on $3.2 \mathrm{~cm}$ centres behind coarse grids on opposite vertical sidewalls of the box. The grids are rapidly retracted (in $\sim 400 \mathrm{~ms}$ ) flush with the sidewalls just before the shock tube is fired. The top and bottom walls of the box are fitted with $15 \mathrm{~cm}$ diameter optical windows for diagnosties, and the vertical upstream and downstream walls of the box are 'shutters', which can be opened rapidly (in $\sim 100 \mathrm{~ms}$ ) just before shock arrival. The operation of the jets, the grids and the shutters is automated in such a way that the arrival time of the shock relative to the decay of the random density field in the box can be varied.

The gas mixture has the same density as air so the mean interface between the scattering region and the uniform gas in the shock tube remains parallel to the plane of the incoming wave during a run. The mean speed of sound of the mixture is also matched to that of air to minimize reflection of the shock from the upsteam and downstream interfaces. In this paper we present results from the study of the 


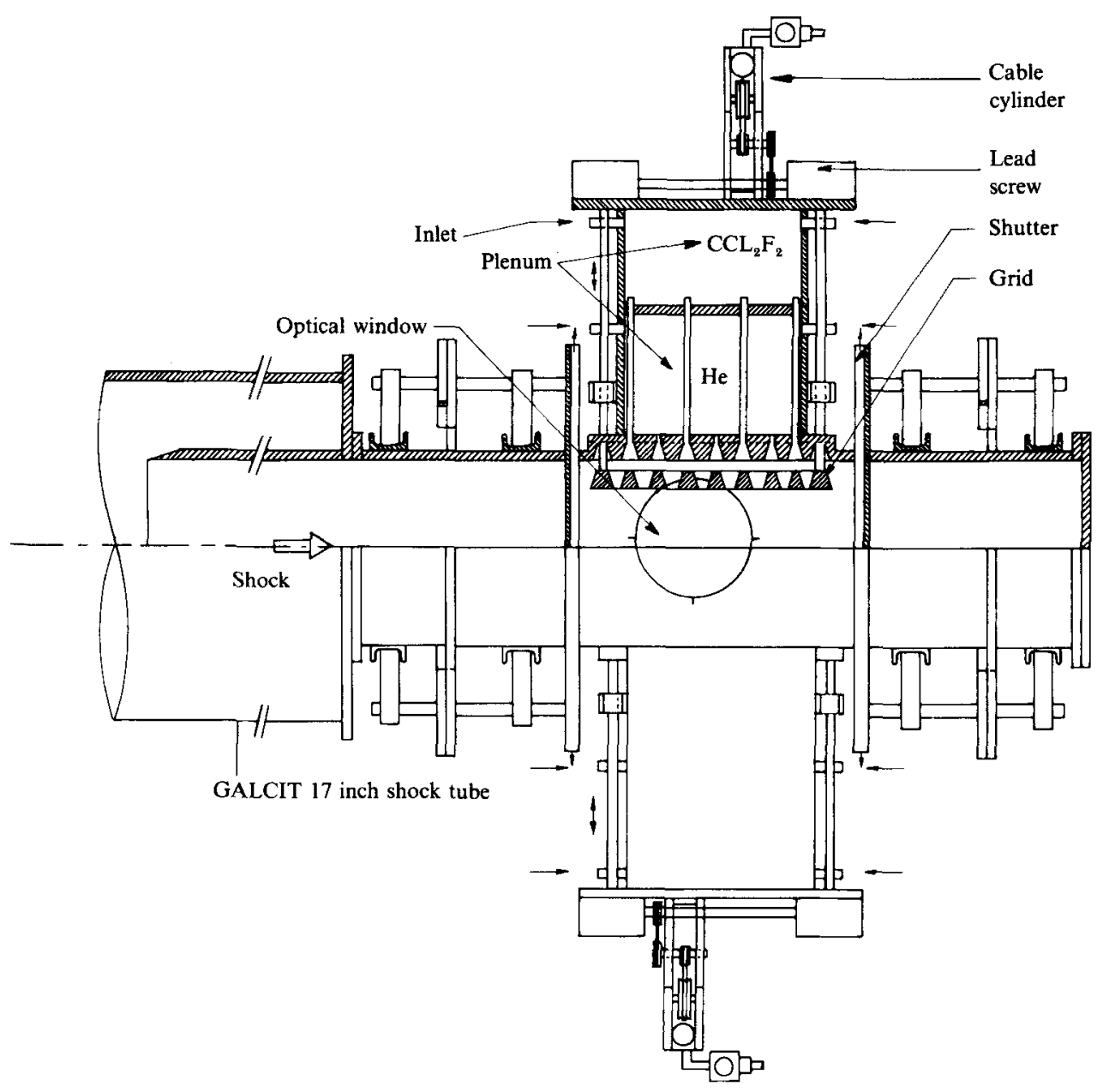

Figure 1. Cross-section and top view of the turbulent-mixer apparatus.

propagation of shock waves of three different strengths $\left(M_{\mathrm{s}}=1.007,1.03\right.$ and 1.1) through the random medium.

\subsection{Experimental procedure}

All mechanical functions of the experiment are controlled by a logic circuit. During a typical run the gas flow to the jets is turned on for $1.5 \mathrm{~s}$, the grids start to retract at $t=1.3 \mathrm{~s}$ and are fully retracted by $t=1.7 \mathrm{~s}$ and the shutters begin to open at $t=$ $1.6 \mathrm{~s}$ and are fully open by $t=1.8 \mathrm{~s}$. The shock is only fired (typically at $t=1.94 \mathrm{~s}$ ) after confirmation that the shutters are fully open. With all this complexity the variation in time of arrival at the test section of the weakest shock waves, $M_{\mathrm{s}}=$ 1.007 , is nevertheless very small, less than $5 \mathrm{~ms}$ in a typical series of runs; the variation in arrival time for the stronger waves is even shorter, typically $1-2 \mathrm{~ms}$.

\subsection{Pressure measurements}

For the purpose of recording the pressure waveform of the perturbed shock wave five piezoelectric transducers are mounted in a movable false endwall downstream of the scattering region. One of the transducers is mounted in the centre of the endwall, and 


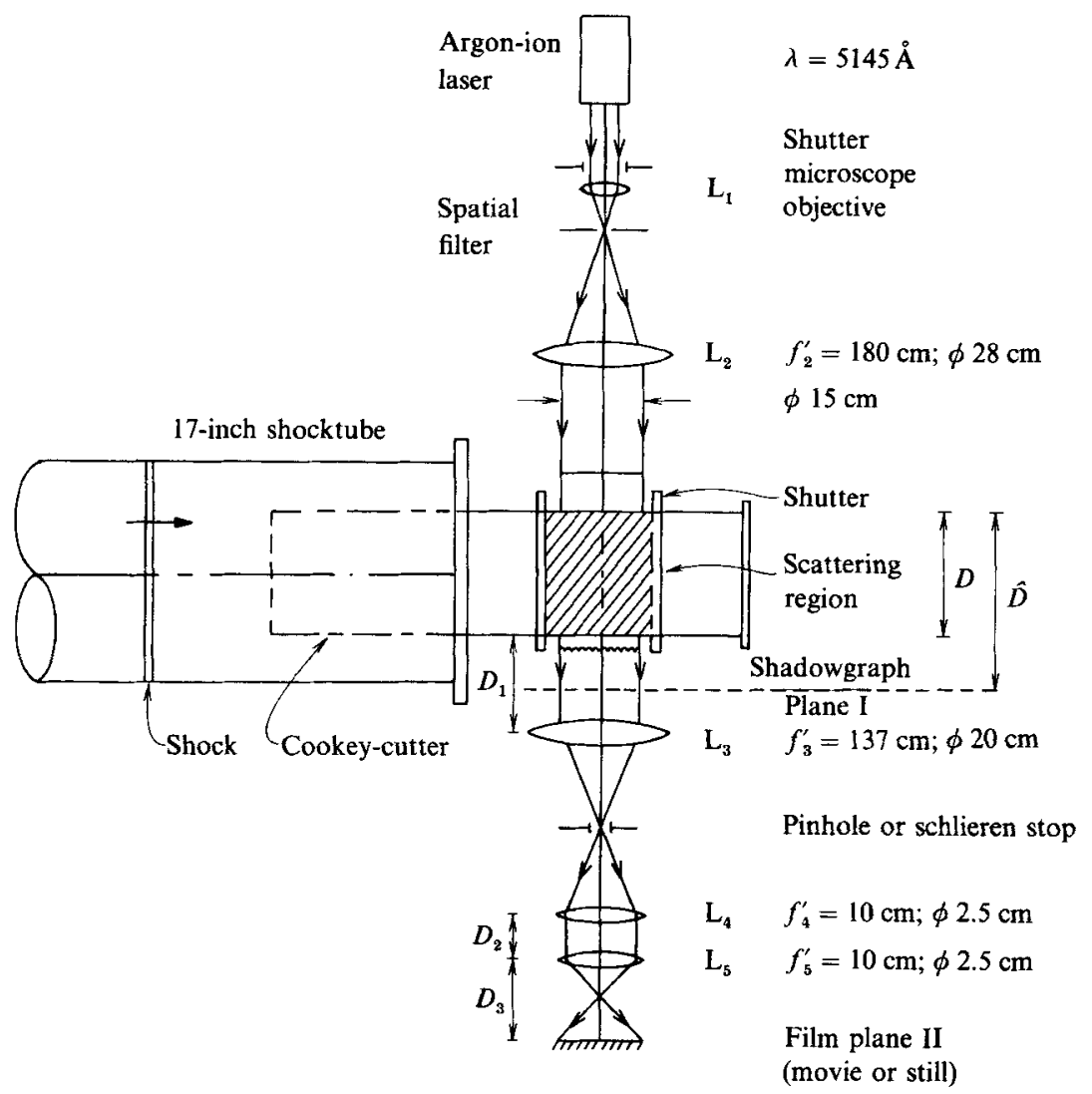

Figure 2. Optical configuration in relation to the shock tube.

the other four are positioned $3.2 \mathrm{~cm}$ radially outward in a cruciform array. The rise time of the transducers is typically $1 \mu \mathrm{s}$. The false endwall can be located either just (i.e. $0.64 \mathrm{~cm}$ ) downstream of the downstream shutter or $21.6 \mathrm{~cm}$ further, near the end of the shock tube. Thus, the effect on the distorted shock wave of propagation through uniform air after emerging from the scattering region can be studied.

\subsection{Optical techniques and digital image processing}

The optical system that was designed and built for obtaining the flow-visualization photographs is shown in figure 2. A beam expander consisting of lenses $L_{1}$ and $L_{2}$ collimates the output of a $4 \mathrm{~W}$ argon-ion laser into a $15 \mathrm{~cm}$ diameter beam. The plane, monochromatic wave of wavelength $\lambda=5145 \AA$ is normally incident upon the random medium. 'The scattered radiation is recorded on film either at the shadowgraph plane I using no imaging optics, or at the film plane II, which is the conjugate plane of the centre of the test section, through three lenses $\mathrm{L}_{3}, \mathrm{~L}_{4}$ and $\mathrm{L}_{5}$. In the back focal plane of lens $\mathrm{L}_{3}$ spatial filtering can be performed by either positioning a pinhole for speckle photography or an opaque dot for schlieren photography ${ }^{+}$.

$\uparrow$ Note that there is no essential optical difference between schlieren and speckle photography, other than the distinction that the former is produced by high-pass filtering and the latter by lowpass filtering of the scattered radiation at the Fourier-transform plane. 
Three types of photographs have been taken during the course of this investigation:

(i) Shadowgraph photographs of the random medium in the absence of the shock wave. An electronic shutter gates the laser output to produce an exposure time of $1 \mathrm{~ms}$.

(ii) Schlieren and shadowgraph still photographs of the shock wave propagating through the random medium. For these photographs the laser is replaced by a spark-gap white-light source that produces a flash of a few microseconds duration.

(iii) Schlieren and shadowgraph movies of the shock wave as it propagates through the medium. The exposure time is set by the operation of a highspeed motion picture camera to be $4.0 \mu \mathrm{s}$ at a framing rate of 50000 f.p.s. or $2.7 \mu \mathrm{s}$ at a framing rate of $75000 \mathrm{f}$.p.s. The spatial resolution of the high-speed camera is 26 line-pairs $/ \mathrm{mm}$ in the direction of rotation of the camera drum and 32 line-pairs $/ \mathrm{mm}$ in the transverse direction on the film.

The negatives of the photographs are digitized on a microdensitometer, and the digital data are stored on magnetic tape for further processing. The results of the processing are displayed on graphs and the digital data can be reconstructed into a photograph using a laser printer.

Information about the statistical properties of the random scattering medium is obtained using the method of Hesselink \& White (1983) for processing images on shadowgraph photographs. A perturbation analysis of the wave equation is used to analyse the data in the limit of vanishingly small optical wavelength and fluctuation amplitude. Under these assumptions geometrical opties theory applies and recourse need not be made to the Born approximation. The spectrum of irradiance fluctuations at some plane downstream of the scattering medium is computed in terms of the scattering parameters. Assuming homogeneity and isotropy, the (two-dimensional) spectrum of the intensity fluctuations in the observation plane is related to the threedimensional spectrum of the index of refraction fluctuations by a Volterra integral equation. The analysis shows that the three-dimensional power spectral density (p.s.d.) $S_{3}(k)$ can be expressed in terms of the two-dimensional p.s.d. of the recorded light intensity variations $S_{I}(k)$ as follows:

$$
S_{3}(k)=\frac{6 \pi S_{I}(k)}{[1+3 \delta(1+\delta)] D^{3} k^{4}}
$$

where $k$ is the magnitude of the wave vector, $\delta=\hat{D} / D, D$ is the thickness of the slab, and $\hat{D}$ is the distance between the entrance plane of the medium and the location of the film plane.

\subsection{Performance of the apparatus and properties of the random medium}

A series of experiments was carried out to define an optimum operating point of the apparatus and to document the properties of the random medium under those conditions. Values of important parameters finally settled upon are as follows: mean concentration of helium by volume $=80 \%$; mean acoustic index of refraction relative to air at standard conditions $=1.00 \pm 0.06$; optical index of refraction $=$ $1.00024 \pm 3.1 \times 10^{-5}$. The above tolerances are the variances of the cited quantities calculated from the measured density fluctuations. 

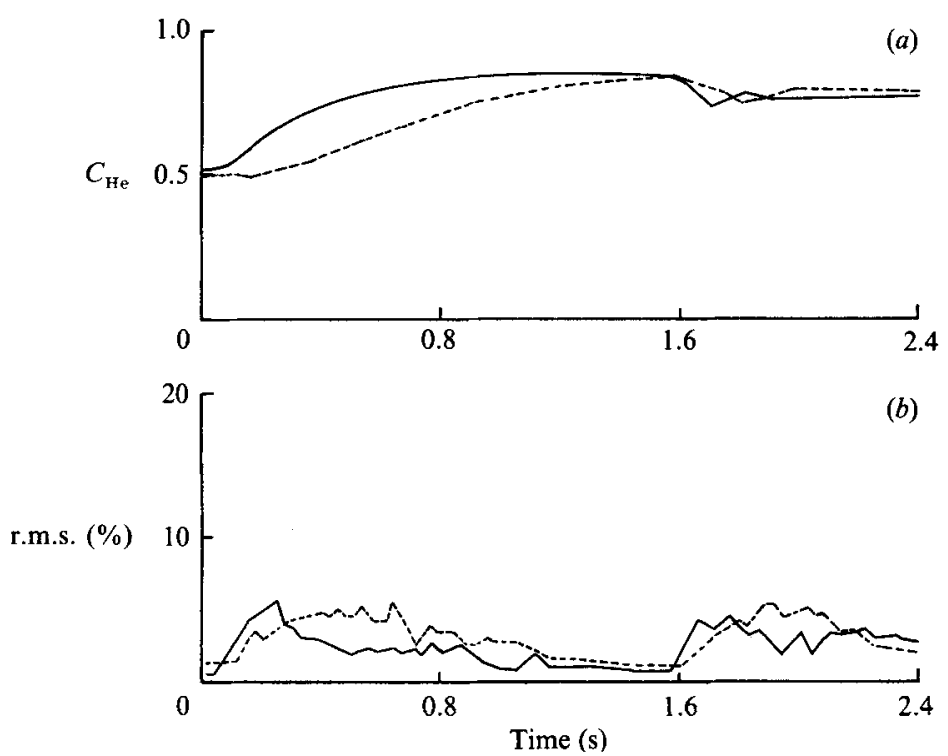

Figure 3. (a) Mean and $(b)$ r.m.s. density profiles measured at the centre of the turbulent medium using an aspirating density probe. The mixture consists of helium and refrigerant 12 . Gas injection into the box is terminated at $t=1.5 \mathrm{~s}$.

\subsubsection{Density probe}

Measurements of mean density and of density fluctuations during the gas-mixing phase of the experiment were made in tests during which no shock waves were fired. An improved version of the density probe of Brown \& Rebollo (1972) was used. Unfortunately, in view of the small mean velocities inside the box in the present experiments, a fixed density probe does not provide as much data describing the statistics of the spatial variation of density as would be the case if gas were being convected past the probe. One of the purposes of the mean-density measurements was to verify that the mixture was homogeneous and free from horizontal or vertical stratification.

Helium concentration measurements. The variation of the helium concentration during the gas-mixing phase of the experiment is shown in figure 3 . The upper plot shows the mean concentration of helium by volume, while the lower plot gives the r.m.s. fluctuation level as a function of time. The solid curves represent data from a probe located $6.6 \mathrm{~cm}$ from a sidewall grid, $12 \mathrm{~cm}$ from the nearest shutter and $13.3 \mathrm{~cm}$ above the bottom of the box. The dashed lines depict data from a probe at the centre of the box, $6.6 \mathrm{~cm}$ above the bottom. Initially, the probe samples air in the test section, and the output voltage of the probe has a value that happens to be about the same as would result from a mixture of $50 \%$ helium and $50 \% \mathrm{R} 12$. Thus, the initial portion of the trace does not represent the true helium concentration and can only be used as an indication of the rapidity with which the air is flushed from the mixing zone. The difference between the data from the two different locations in the box is explained by the fact that the steady-state concentration level is reached much earlier near the grids than in the middle of the box. An amount of gas equal to about four times the volume of the test section is needed to completely flush out the air and to reach a steady state.

The equilibrium state is perturbed when the jets are turned off (at $t=1.5 \mathrm{~s}$ ). At 


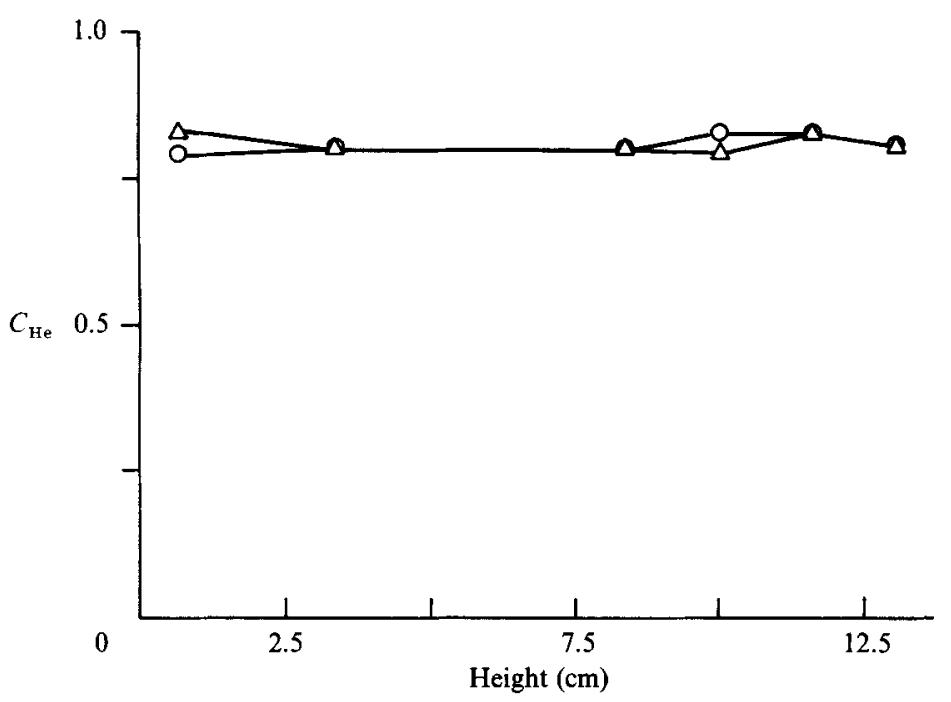

Figure 4. Mean density measurements as a function of the height above the bottom of the turbulent mixer.

that instant, both plena store the same volume of gas, but the pressure in the R12 tank is higher than in the helium reservoir, yet the helium exhausts at a faster rate. Consequently, the helium content of the injected gases decreases as time increases, and after the jet flow terminates a new plateau is reached. Later, at $t=2.5 \mathrm{~s}$, the concentration drops again because, after the shutters open, air eventually permeates into the box.

The data for r.m.s. fluctuation shown in figure $3(b)$ are influenced by the same changes of operating conditions. The first increase is associated with removal of air from the box. Then, the magnitude of the r.m.s. fluctuation decreases monotonically until a steady value is reached. The low level of fluctuations during this second phase suggests that the turbulent mixing process is very efficient. After the jets are turned off, the r.m.s. fluctuations again increase, mainly because the concentration of helium decreases with increasing time until the plena are depleted. During the final stages of the injection phase almost pure R12 at very low velocity flows through the jets. In fact, this behaviour suggests an alternative operating scheme to increase the r.m.s. fluctuations: the test section could first be filled with pure helium and then R12 could be injected for such a length of time that, after the jets are turned off, the average concentration is $80 \%$ helium by volume. However, in this method the flowcontrol system could not be tuned by the scheme to be described later in this section. Figure 4 shows the vertical profile of helium concentration at the centre of the box, obtined by averaging the data from five runs at each measuring station. The observed $2 \%$ variation in the mean value is within the experimental accuracy.

After the shutters open, the air outside the test section begins to diffuse into the test section by turbulent mixing enhanced by the wakes of the opening shutters. The width of the resulting interface has been estimated by comparing the signal from one probe near the centre of the box with the signal from a probe positioned very close to a shutter, in this case both probes were located equidistant from the two sidewalls containing the jets and at the same height above the bottom wall. It was found that the transition region extends approximately $7 \mathrm{~cm}$ into the test section when the shock is fired. However, since on the interface both mean density and speed of sound 
are matched, the transition region has little effect on the shock wave other than to initiate the scattering process.

Determination of large lengthscales. A pair of density probes were used to obtain two-point correlation functions for the density fluctuations. For each separation of the probes the parameters of the correlation function, assumed to be Gaussian, were computed by averaging the results of 16 runs. From the correlation function, the integral scale $L_{\rho}$ was determined to be approximately $1.5 \mathrm{~cm}$. The rather large scatter in the data, caused by the small convection velocites in the apparatus, precluded the determination of the actual shape of the correlation function.

Final tuning of the apparatus. The procedure for final testing of the performance of the turbulent mixer involves both density-probe measurements and pressure measurements. With one probe located near each sidewall containing the jets, five runs are made and the average density is calculated. The settings of the gas supply system are altered until both probes indicate the same mean concentration of helium to within $\pm 1 \%$. The probes are then removed, and a further test of the properties of the medium is performed by running the full system as in a normal run except that a $2.4 \mathrm{~s}$ delay is introduced to allow complete mixing in the test section before the shock wave is fired. The settings of the gas-flow control system are then adjusted before repeated shock tube runs until the pressure signals from the transducers in the false endwall indicate that the incident shock wave is not perturbed in any way. This turns out to be a very stringent test, for it verifies that both the mean speed of sound and the acoustic impedance of the mixture are equal to those of the air in the shock tube.

Pressure signals recorded after making the final adjustment are shown in figure 5 . The oscillogram $(a)$ displays two pressure signals which are simultaneously measured in the false endwall during one run in which there is a long delay before the shock tube is fired. The gauges are located $6 \mathrm{~cm}$ apart and the shock Mach number is $M_{\mathrm{s}}=1.03$. The observed pressure histories are typical of unperturbed shocks. In particular, the high-frequency fluctuations immediately behind the front are due to ringing of the transducers caused by the steep-fronted shock. The lower-frequency variations of pressure on the traces may be due to diffracted waves from small gaps that exist between the shutter blades and the sidewalls of the test section. It is concluded that neither the mean properties of the scattering medium nor the interface between the quiescent air in the shock tube and the helium-R12 mixture significantly modify the incident shock wave.

For comparison, the effect of the turbulence on the incoming, wave in a run in which the long delay is not introduced is shown figure $5(b)$. The signals are measured with the same transducers and at the same measuring stations as above. The top trace shows that the shock strength has been increased above that of the incident shock and that the initial disturbance is followed first by an expansion and then by a slow compression. The bottom trace has a smoother rise and does not show any pressure overshoot, the leading disturbance being followed by an increase of the pressure toward the unperturbed value. Thus, it is evident that the pressure signature of a scattered wave can be very different at different locations on the wave front.

\subsubsection{Image processing: small lengthscales}

To test the method described in $\$ 2.4$ for determining the power-spectral-density function of the random medium, the spectrum of the light intensity fluctuations that are recorded on film has been computed and averaged from the digitized images of 


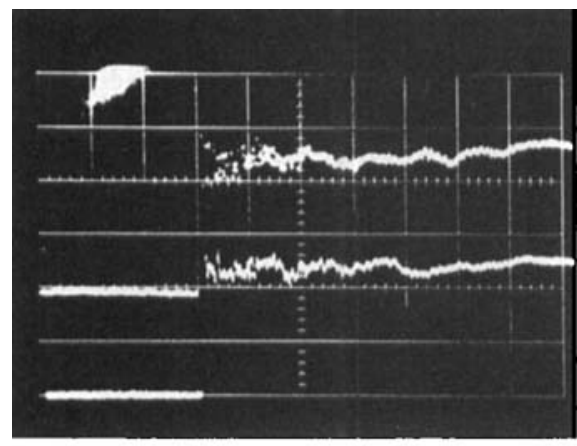

(a)

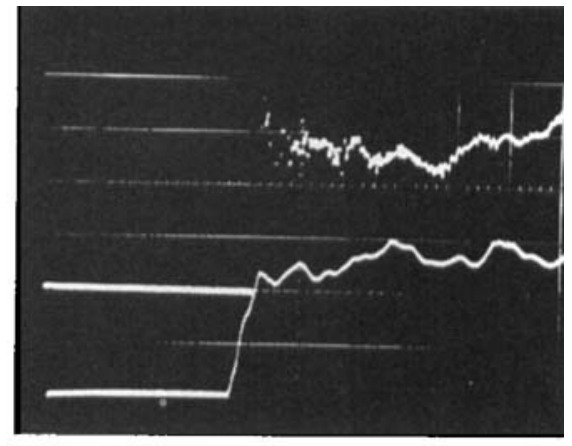

(b)

Figure 5. Pressure profiles of shocks ( $a$ ) after turbulence decay and $(b)$ with turbulence. The two traces are measured simultaneously at different locations on the shock front. The horizontal timescale is $20 \mu \mathrm{s} /$ div; vertical scale is 1.5 p.s.i. $/ \mathrm{cm}$.

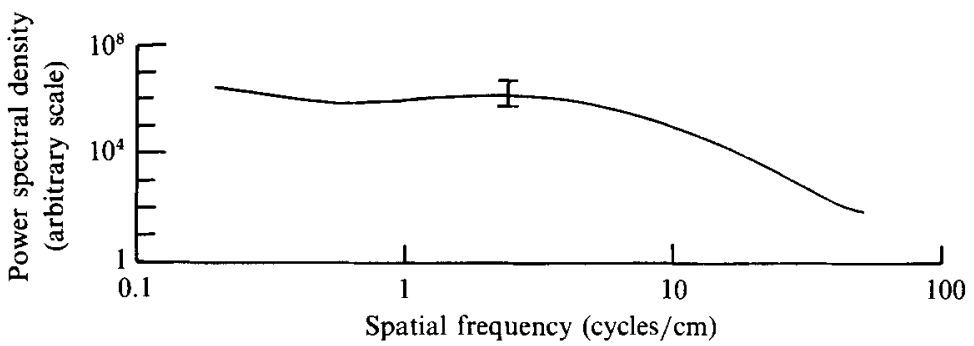

Figure 6. Power-spectral-density curve for intensity fluctuations recorded on film using the shadowgraph method.

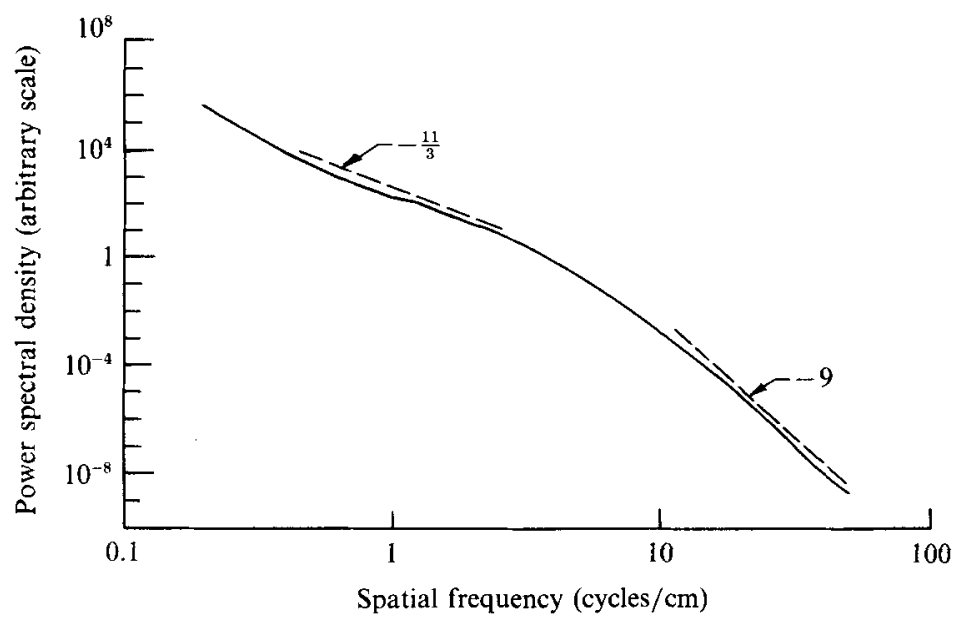

Figure 7. Three-dimensional power-spectral-density curve of concentration fluctuations in the random medium.

eleven still photographs of the medium unperturbed by the passage of a shock wave (figure 6). Care is taken to limit the dynamic range of the intensity fluctuations so that the response of the photographic film is linear in the range of spatial scales of interest in this work $(\sim 0.05-2.0 \mathrm{~cm})$. The standard deviation is indicated by the error bar in the figure. Then, from the two-dimensional power spectrum of the image 
the three-dimensional spectrum of the medium is calculated using equation (1) (figure 7). For comparison, two lines with slopes $-11 / 3$ and $\mathbf{- 9}$ are shown on figure 7. According to Kolmogorov similarity, in homogeneous turbulence the p.s.d. (the spectrum function) in the inertial subrange varies as the $-11 / 3$ power, while in the viscous subrange the Heisenburg spectrum, supported to a certain extent by experiment, suggests that the spectrum should vary approximately as the $\mathbf{- 9}$ power (Townsend 1976). In the figure the transition from the $-11 / 3$ power to the -9 power occurs at a wavenumber $k=5.3$ cycles $/ \mathrm{cm}=33.3 \mathrm{rad} / \mathrm{cm}$, which corresponds to a lengthscale of $1.9 \mathrm{~mm}$. Taking this length to be the Kolmogorov microscale $\gamma_{\rho}$, it can be seen that in the turbulence studied in the present experiments there is about one order-of-magnitude separation between the microscale and the integral scale.

It is in principle straightforward to compute the correlation function from the three-dimensional p.s.d. using Fourier-transform techniques. However, a difficulty arises. The p.s.d. function for small wavenumber is not accurately known, because in this range the sampling is inadequate owing to the finite size of the photograph. Therefore, the asymptotic behaviour of the correlation function is uncertain, and accurate results cannot be obtained. An attempt to compute the correlation function of the medium directly from the correlation function of the photograph also produces unreliable results (Hesselink \& White 1983) for essentially the same reason. The accuracy of the data associated with the large-scale features can only be improved by substantially increasing the population used for the ensemble averages.

\section{Experimental results}

\subsection{Photographs}

Both schlieren and shadowgraph techniques were used to visualize the shock waves and the random medium in these experiments. The two methods emphasize different aspects of the phenomenon. The schlieren system brings out shock-wave topography much more clearly than the shadowgraph pictures, presumably due to its greater overall sensitivity, while the shadowgraph emphasizes the small scales in the random medium and is more amenable to quantitative treatment. However, neither the schlieren nor the shadowgraph system actually used was sensitive enough to detect the weakest shock waves studied $\left(M_{\mathrm{s}}=1.007\right)$, though such shocks were easily visible in a uniform (non-scattering) medium. On the other hand, waves of strength $M_{\mathrm{s}}=1.03$ and 1.1 could conveniently be visualized by these methods; figures 8 and 9 show schlieren photographs taken with spark flash illumination of such shocks propagating through the shock-tube test section. Typical shadowgraph photographs are shown in figure 10. Finally, figures 11 and 12 show sequences of frames from schlieren and shadowgraph movies, respectively, of shocks propagating through the random medium. These figures do not show the detail of the still pictures because the motion-picture camera has much lower spatial resolution. In all photographs the diameter of the viewing window is $15 \mathrm{~cm}$.

It is clear from all the photographs that propagation through the random medium has strongly modified the wave front, which in a uniform gas appears thin and straight. In the schlieren photographs and, to a certain extent in the shadowgraph photographs, the shock wave appears to have been broken up into a succession of fronts which fall within a region of approximately $1 \mathrm{~cm}$ thickness. However, because schlieren and shadowgraph photography integrate across the width of the test section, another possible interpretation of the striations observed on the front, an interpretation which has, in fact, been proposed by some authors, is that they 


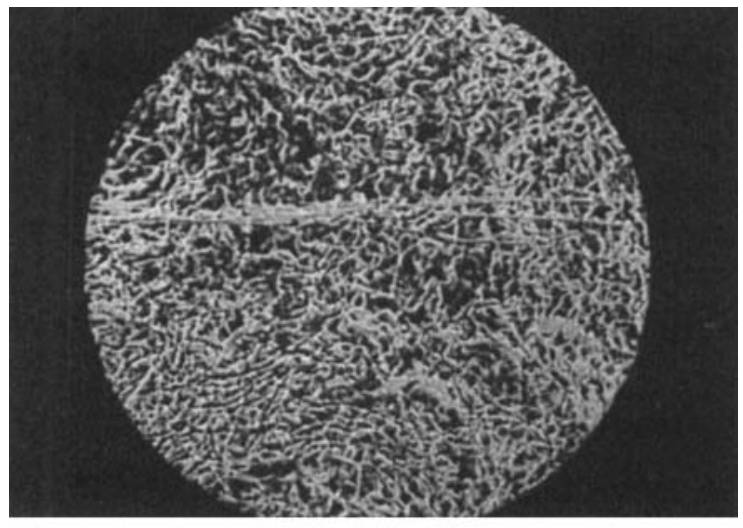

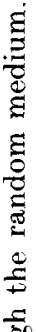

$\frac{80}{3}$

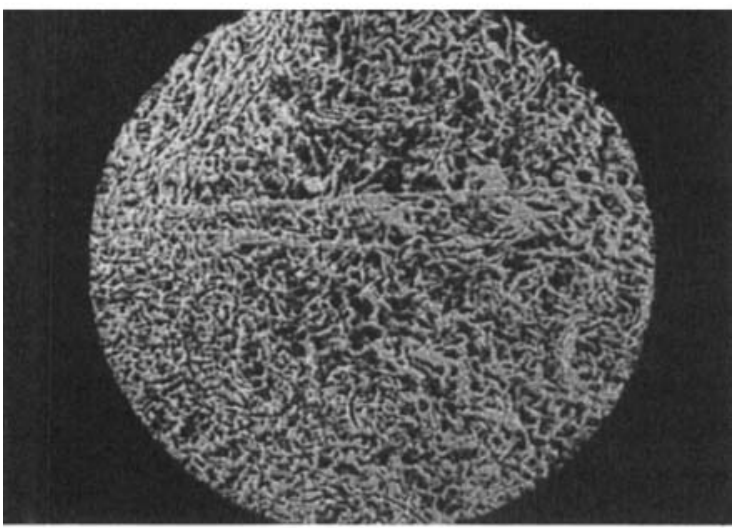

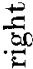

$\circ$

$\stackrel{+}{4}$

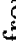

3

峁

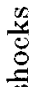

$\infty$

$\stackrel{8}{-}$

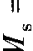

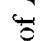

蛋 

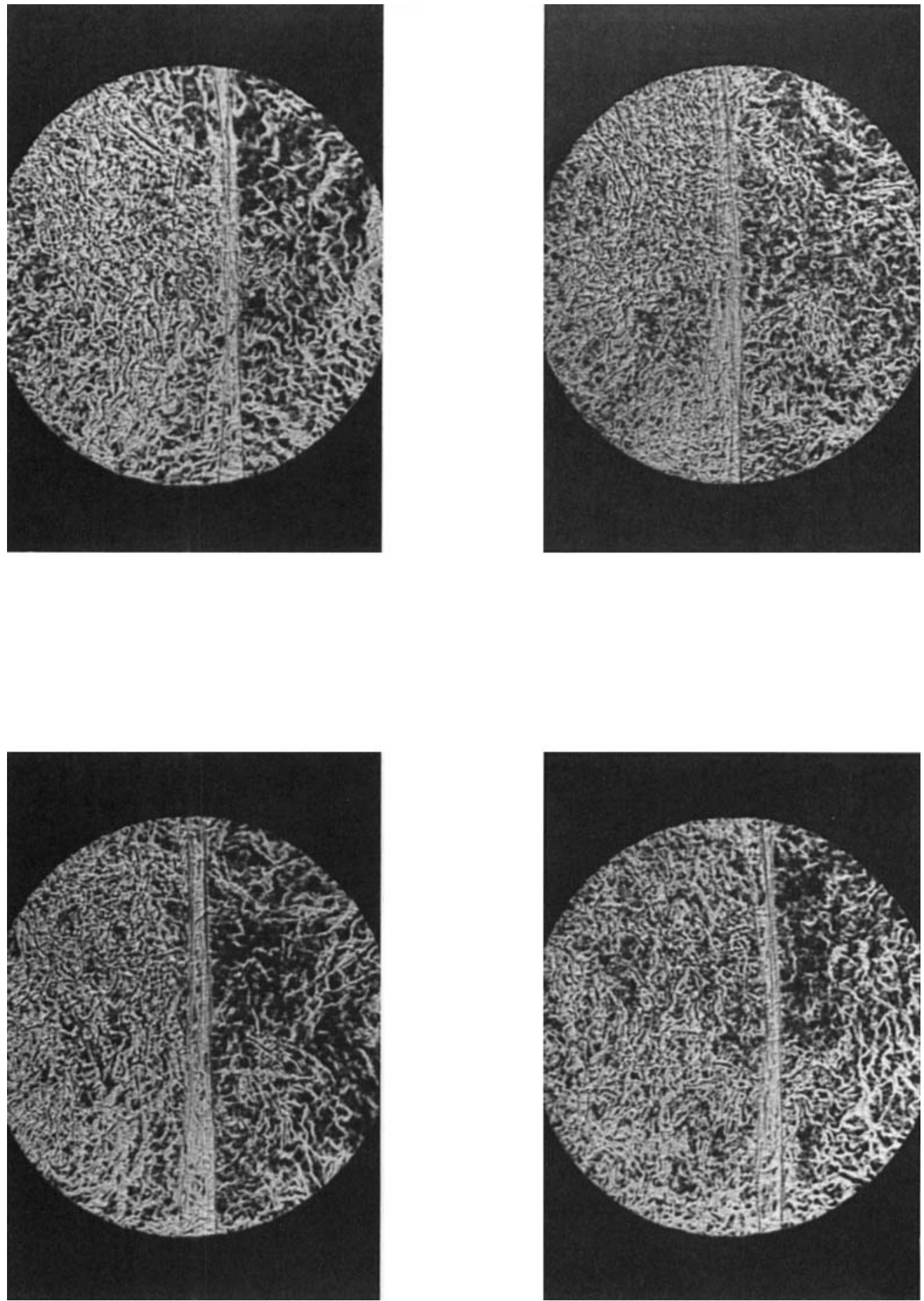

FIGURE 9. Schlieren photographs of $M_{\mathrm{s}}=1.1$ shocks moving from left to right through the random medium. 


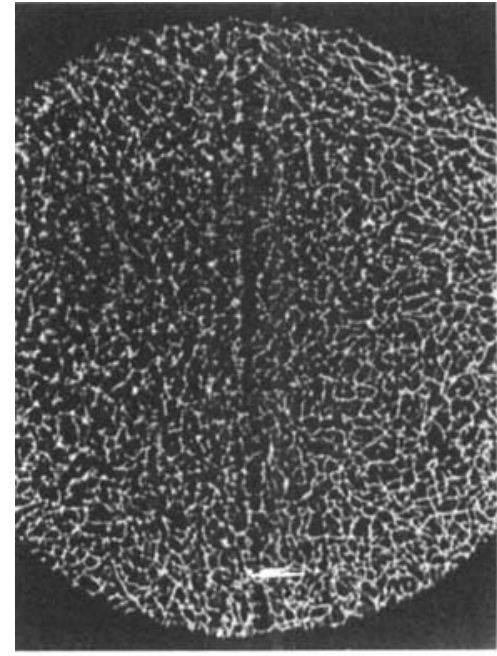

$M_{\mathrm{s}}=1.03$

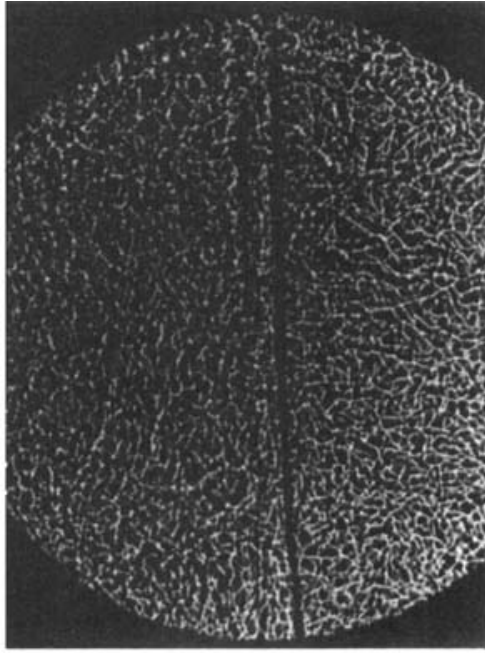

1.1

Figure 10. Shadowgraph photographs of shocks moving from left to right through the random medium.

represent the maxima and minima of wrinklest on a single wave front. However, if this were the case, since the randomness is distributed isotropically in the medium, wrinkles of the same amplitude would be seen in profile on both the leading and subsequent fronts.

It was shown analytically by Kulkarny \& White (1982), and confirmed by the numerical ray-tracing calculations of $\S 4$, that even when the amplitude of the soundspeed fluctuations is small, the tendency to focus is so strong that substantial wave folding results. Calculations on geometrical acoustics show that, under conditions typical not only of the present experiments but also of sonic booms from supersonic transports propagating through the terrestrial atmosphere's turbulent boundary layer, focusing occurs. Therefore the proper interpretation of the image of the scattered field in figures 8-12 is that it represents a multiplicity of wave fronts, rather than a single, wrinkled front. This conclusion is further confirmed by the measured pressure histories (cf. $\$ 3.2$ ) in which the pressure closely behind the initial disturbance fluctuates with rather large amplitude, suggesting the presence of scattered waves. Consequently, for the purpose of this paper we adopt the view that the effects of the random medium on the shock front can best be considered in terms of random focusing and defocusing of the front by the inhomogeneities in the medium, which constitute a random array of lenses (Pierce 1968) and that, in the present experiments, the refraction is sufficiently strong that the wave fronts become multiply folded.

The photographs of figures 8-12 (especially the schlieren photos) show that the fluid in the test section has also been modified by the passage of the shock wave. In particular, the large number of bright lines which appear in the shocked fluid to the left of the wave fronts in figure 9 are remarkable. These features seem to be oriented preferentially in the direction parallel to the wave front, giving the impression that

$\dagger$ In this paper we use the term wrinkle to describe relatively short-wavelength distortions of a wave front, such that many wrinkles would be visible on a given front in the test section. 


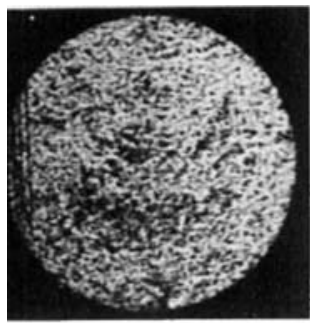

$t=20 \mu \mathrm{s}$

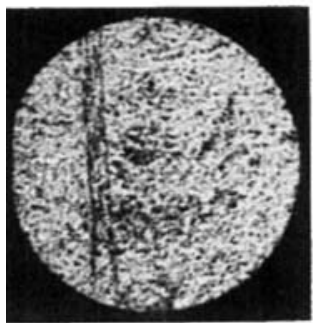

120

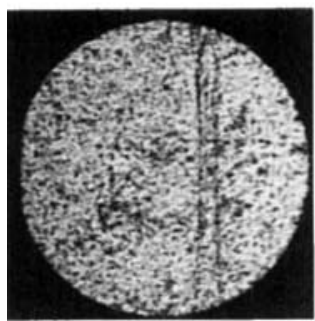

Thก

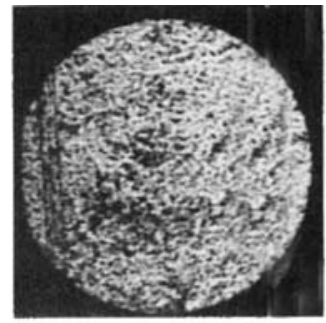

40

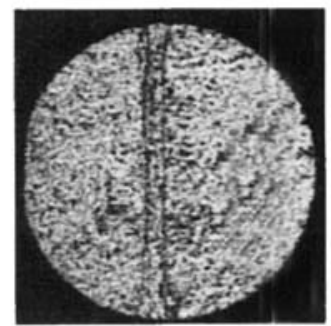

180

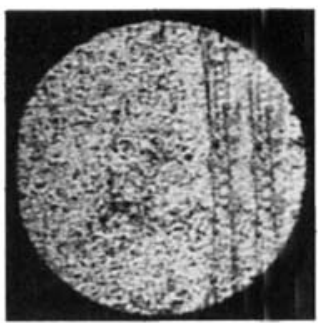

280

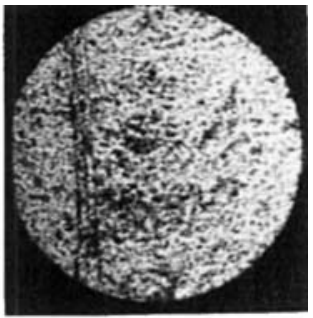

100

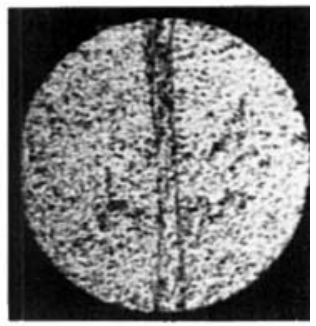

200

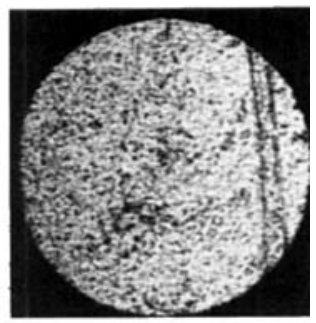

340

Figure 11. Schlieren movie of $M_{\mathrm{s}}=1.03$ shocks moving from left to right through the random medium.

a rather substantial compression of the upstream fluid has occurred perpendicular to the plane of the shock wave, together with a corresponding elongation parallel to the shock wave. Indeed, the apparent compression is far greater than that which would be calculated from the shock jump conditions. This effect, which is probably enhanced by the formation of caustics in the refracted optical field at the film plane, is not understood.

\subsection{Pressure field}

The pressure measurements exhibit the nature of the initial disturbance and record the scattered field behind the main front. A major consequence of focusing in the random medium, if it occurs, is that not all of the wave fronts in the scattered field will remain discontinuous in amplitude, because acoustic fronts that have passed through one or more foci are only discontinuous in the first or higher derivatives 


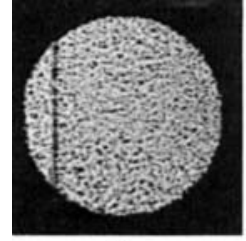

$t=60 \mu \mathrm{s}$

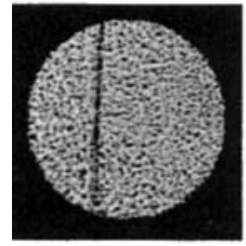

140

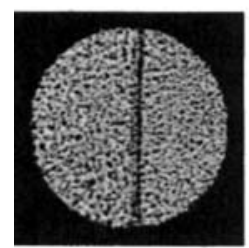

220

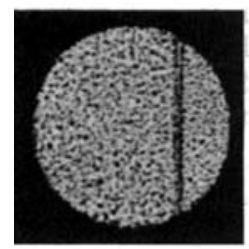

300

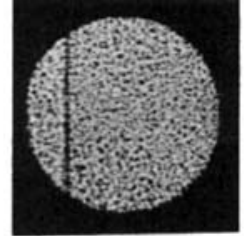

80

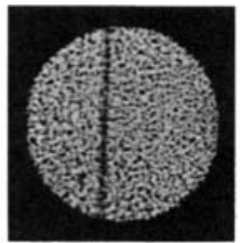

160

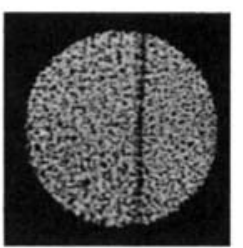

240

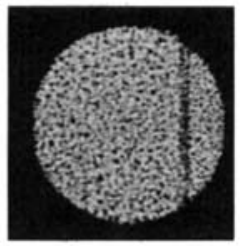

320

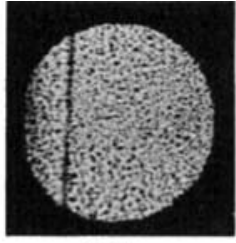

100

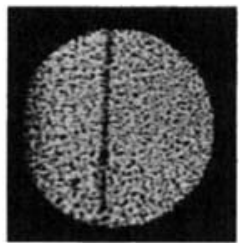

180

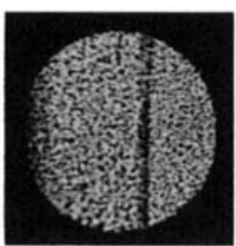

260

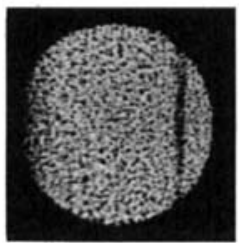

340

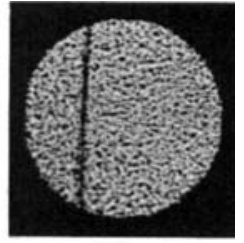

120

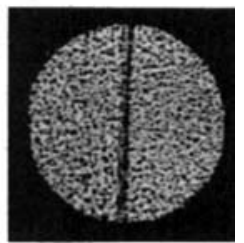

200

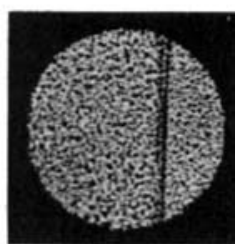

280

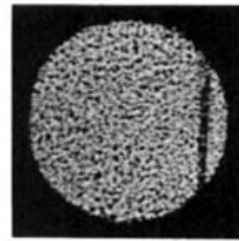

360

Figure 12. Shadowgraph movie of $M_{\mathrm{s}}=1.03$ shocks moving from left to right through the random medium.

(Friedlander 1958). Consequently, scattered and focused fronts may be difficult to detect.

\subsubsection{General characteristics of the pressure signals}

A representative series of oscillograms recorded simultaneously by four different transducers is shown in figure 13 for the three shock strengths, $M_{\mathrm{s}}=1.007,1.03$ and 1.1. For the $M_{\mathrm{s}}=1.007$ case, trace 1 shows three discrete pressure jumps in the initial rise which are clearly caused by a succession of three shocks. Similarly, the front on trace 3 may be composed of two jumps, and the front on trace 4 shows three jumps, but their amplitudes are small, so they are somewhat difficult to distinguish. Thus it frequently happens that the weak shock waves show relatively slow rise times, apparently because they are made up of a succession of weaker fronts. 

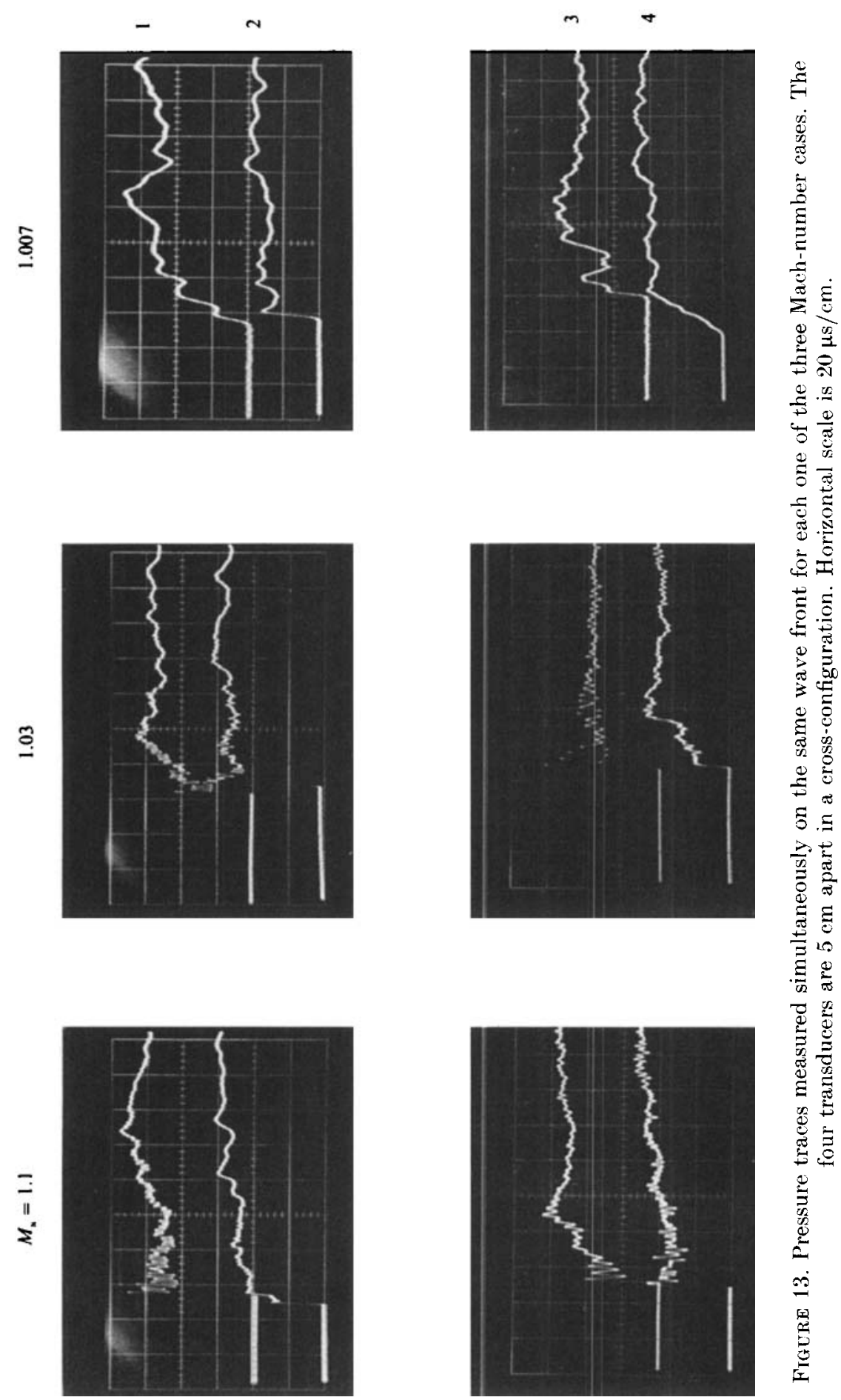

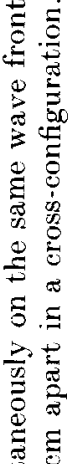

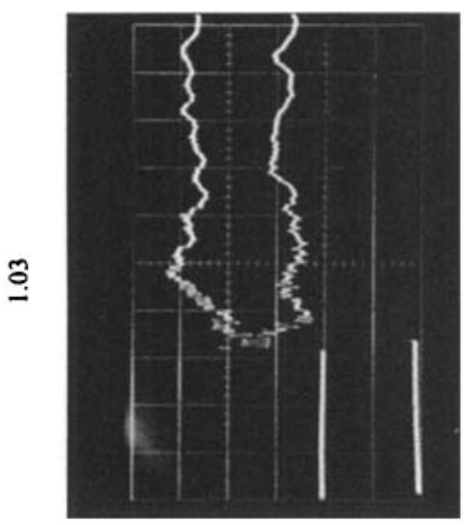

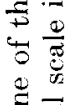

$\div$

可

오

10

然

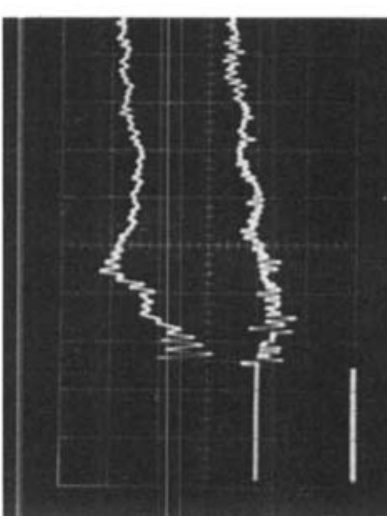

年 

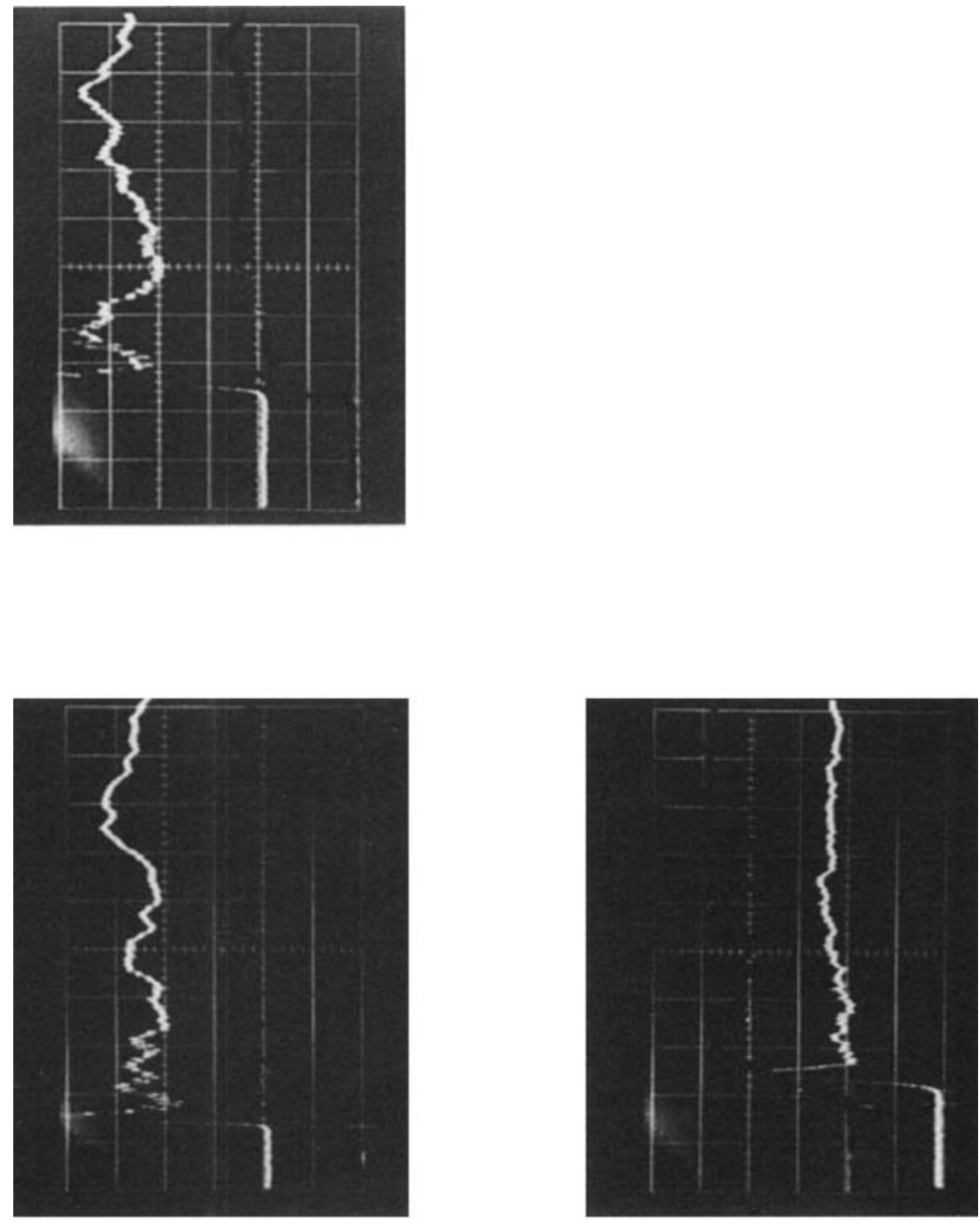

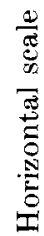

ב

$\stackrel{80}{\Xi}$

ב

憵

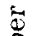

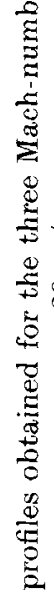

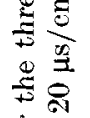

$\stackrel{\Xi}{5}$
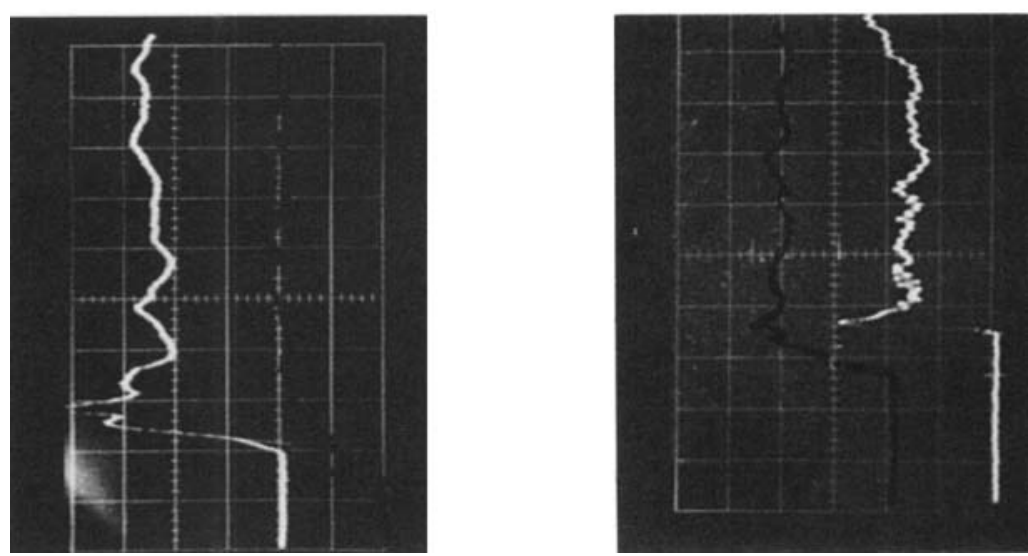

 

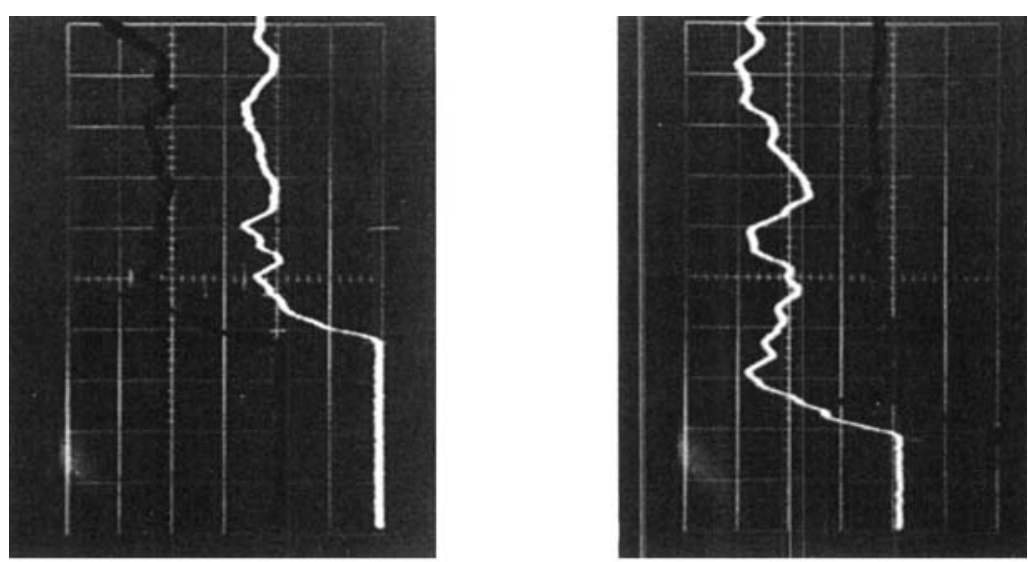

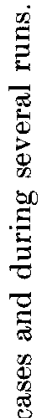
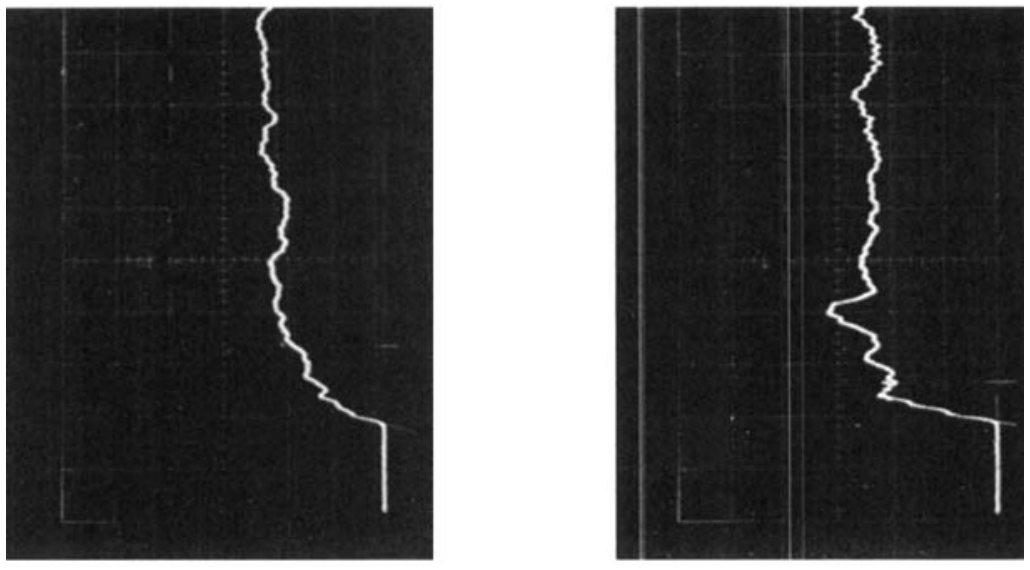

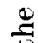

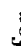

.

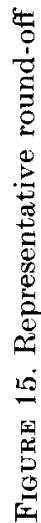
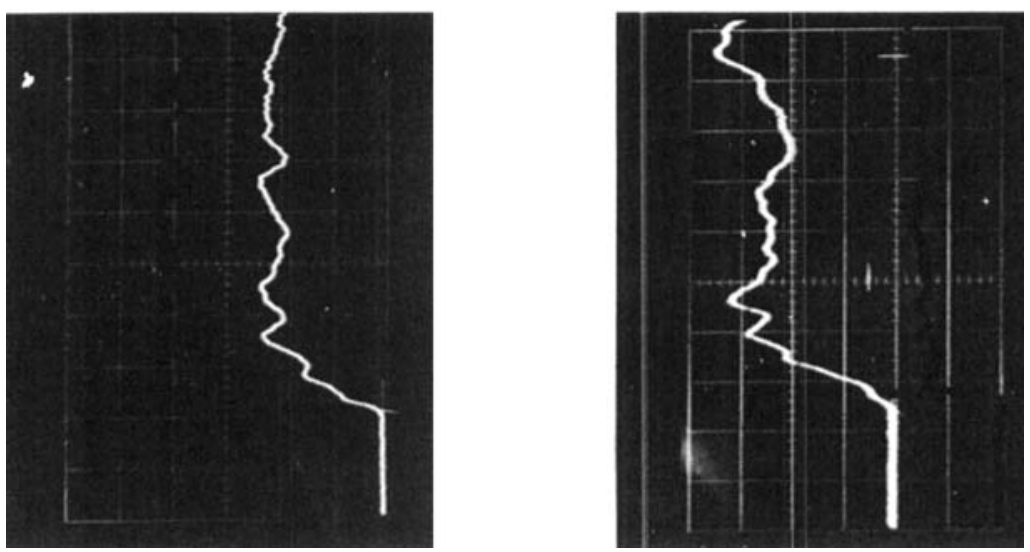

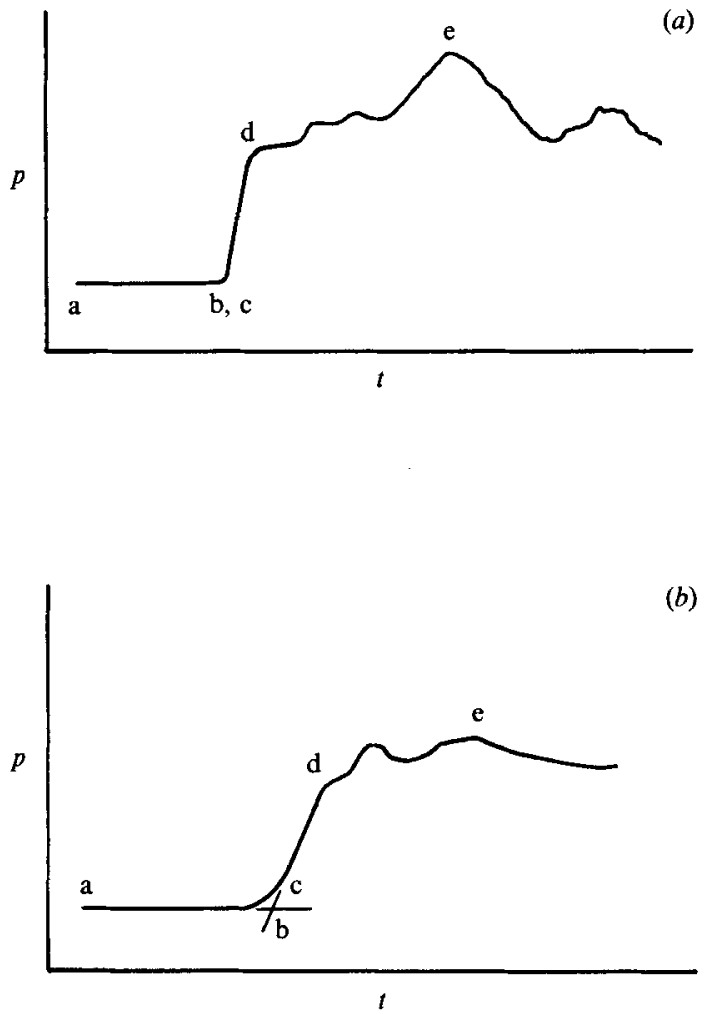

Figure 16. Schematic pressure profiles showing $(a)$ no precursor and $(b)$ a precursor. The letters a-e indicate significant points on the traces, described in \$3.2.1.

Another important observation is that all 128 pressure traces for the $M_{\mathrm{s}}=1.007$ shocks depart from the baseline much more gradually than for the stronger shocks, in an upward-curving trace that begins as much as $2-4 \mu$ s ahead of the main disturbance, a feature we have labelled precursor. In figures 14 and 15 , representative examples are presented of pressure histories observed after a $M_{\mathrm{s}}=1.007$ shock wave has propagated through the volume of turbulence, showing traces that are especially sharp or peaked and traces with particularly slow rise times, respectively.

An alternative explanation of the long rise times of the shocks arises from the fact that a gas with a structurally complicated molecule is used as the test medium, so the relaxation of molecular vibrational degrees of freedom might be greatly different than for, say, a monatomic gas, and the shock front is consequently substantially dispersed. $\uparrow$ However, this possibility can be eliminated in the case of R12 because the appropriate relaxation times at atmospheric pressure are much shorter even than the time response of the pressure transducers.

Stronger shock waves are less affected by the scattering properties of a random medium than are weak shocks. Nevertheless, the pressure histories of the $M_{\mathrm{s}}=1.03$ and 1.1 shocks (figure 13) exhibit many interesting features. The second trace of the $M_{\mathrm{s}}=1.03$ case is noteworthy because it shows a sharp maximum just behind the wave front, followed by an expansion. This is referred to as a peaked waveform. Also,

$\dagger$ The authors are grateful to M. J. Lighthill for calling this to our attention in connection with early experiments with $\mathrm{CO}_{2}$ (Hesselink 1976). 
the shock arrival times documented in figure 13 show variations from one transducer to another of order $5 \mu \mathrm{s}$.

The traces for the $M_{\mathrm{s}}=1.1$ shocks initially rise sharply; no precursor signal has been observed on any of the $M_{\mathrm{s}}=1.1$ traces. Furthermore, the magnitude of the pressure fluctuations behind the waves are substantially reduced. The high-frequency fluctuations evident on the strong-shock pressure traces is due to transducer ringing (cf. $§ 2.5 .1$ ). The second trace of the $M_{\mathrm{s}}=1.1$ case shows a distinct but weaker second front behind the main disturbance.

The important features of the pressure traces are shown schematically in figure $\mathbf{1 6}$. Two typical cases are shown : in figure $\mathbf{1 6}(a)$ the main disturbance is relatively sharp while in figure $16(b)$ it is preceded by a precursor. The distance $a-b$ in the figure defines the so-called 'arrival time' of the wave front, measured from the time at which the three recording oscilloscopes are simultaneously triggered. The time between points $c$ and $d$ will be called the 'shock' or 'wave-front rise time'. The pressure corresponding to the vertical distance between $c$ and $d$ will be called the 'shock pressure'. The 'peak pressure' of the trace occurs at the point e, and the time lag between the main front and the peak pressure, called the 'time interval to peak pressure', is defined as the time from d to e. Any separation between points $b$ and c signifies the occurrence of a 'precursor'. The shock and peak pressures are normalized with the unperturbed shock strength.

The features of the pressure traces described above are all remarkably similar to those previously identified during early flight tests examining the sonic boom (Maglieri \& Parrott 1963). In those tests both 'peaked' wave forms with substantial overshoot and 'rounded' signatures with abnormally long rise times were recorded. The present laboratory-generated traces show that slow rises, which one might describe in simple terms as rounded, are often actually made up of a series of small steps. As in the flight tests, both the peaked and rounded forms of pressure traces occur in our experiments frequently but randomly, in addition to traces with more conventional-looking shock discontinuities such as, for example, trace 4 of the $M_{\mathrm{s}}=$ 1.1 case and trace 3 of the $M_{\mathrm{s}}=1.03$ case in figure 13. Rather extreme examples of peaked and rounded pressure histories are often seen simultaneously even at adjacent pressure transducers in the same experiment, i.e. in close proximity on the same shock front. Crow (1969) showed from considerations of geometrical acoustics why such behaviour is to be expected.

The qualitative nature of the pressure traces may be more clearly understood in terms of the tendency of a random medium to randomly focus and defocus propagating waves. It is known (Sturtevant \& Kulkarny 1976) that, depending on the strength of the incident shock wave, the intensity of the perturbations of fluid properties causing focusing and the size of the focusing elements, the wave fronts may or may not subsequently cross and fold. Folded wave fronts typically show a slow initial pressure rise, similar to the property we have labelled precursor, followed by a more rapid increase in the primary (focused) front (Friedlander 1958). On the other hand, unfolded wave fronts near foci typically exhibit a sharply peaked pressure signature with a rapid expansion following immediately behind the primary front. Trace 2 of the $M_{\mathrm{s}}=1.03$ case, figure 13, is representative of this behaviour.

\subsubsection{Wave-front rise time: $M_{\mathrm{s}}=1.007$}

The results of the measurements of the rise time of the first major disturbance on the weakest shock waves studied in these expriments are shown in figure 17. The minimum value is limited to $1 \mu \mathrm{s}$ by the response of the pressure gauge. The stronger 

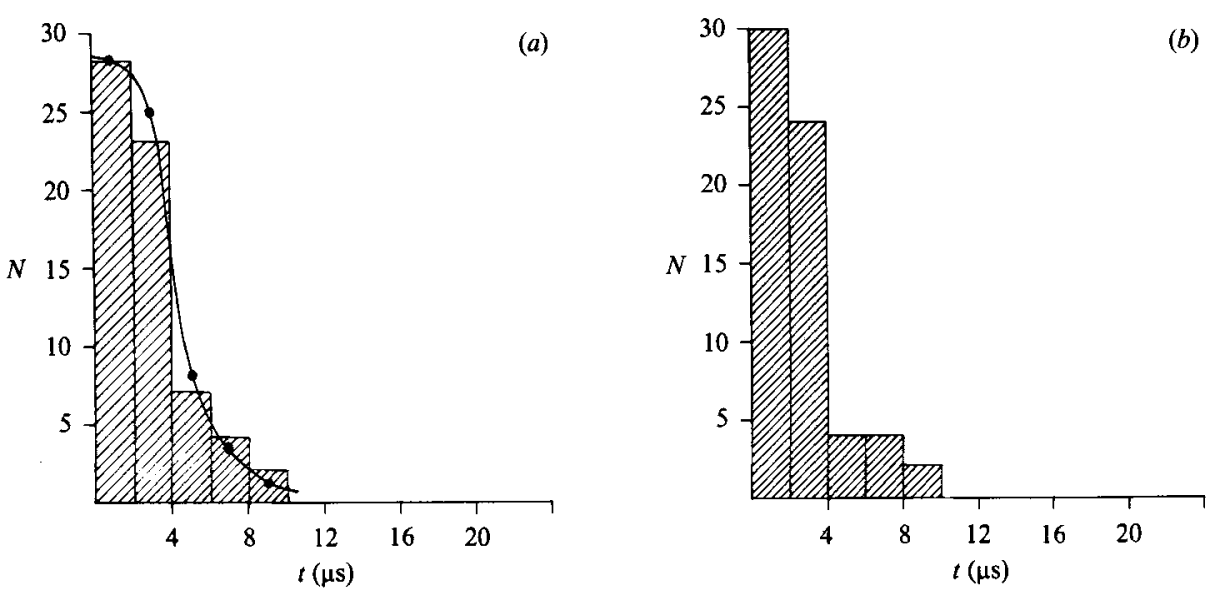

Figure 17. Histograms of shock rise time measured at $(a)$ the exit shutter or at $(b)$ the downstream station; dots connected by line denote results from numerical ray-tracing calculations.

shock waves all show rise times within the resolution of the transducers. The rise time implied by the calculated Taylor thickness of the $M_{\mathrm{s}}=1.007$ shock wave is approximately $0.1 \mu \mathrm{s}$, so measured rise times of more than $1 \mu \mathrm{s}$ imply an increase by scattering of the thickness of the incident wave by as much as one order of magnitude. Figure $17(a)$ shows that, in fact, in 13 out of 64 of the traces recorded near the downstream boundary of the scattering medium, rise times from 4 to $10 \mu \mathrm{s}$ were observed in these experiments. Another remarkable observation which will be discussed below is the similarity between the distribution of rise times immediately downstream of the scattering medium (figure $17 a$ ) and after $22 \mathrm{~cm}$ of clear-air prop gation (figure $17 b$ ).

W $\_$now consider the possibility that tilting or wrinkling of an otherwise single, thin shock front could cause an apparent thickening of the initial front. In order that large-scale and/or small-scale distortions (i.e. tilt or wrinkling, respectively) of a single front might cause the observed rise times, they must be of large enough amplitude that the reflection of the distorted front from the face of the $5 \mathrm{~mm}$ dia. transducer takes 4-10 $\mathrm{s}$. One measure of tilt on a $6.3 \mathrm{~cm}$ scale (the separation between the transducers on the false endwall) can be obtained from the measurements of the mean difference of arrival times at pairs of transducers (cf. §3.2.4), the largest value of which is $14.9 \mu \mathrm{s}$. It implies that the mean rise time caused by distortions of this scale on a shock front arriving at a $5 \mathrm{~mm}$ diameter transducer cannot be more than $1.2 \mu \mathrm{s}$. However, occasionally, individual differences of arrival time can be somewhat larger than the mean value, and the most tilted portion of the front could be just that portion which actually touches the transducer. In fact, the maximum observed arrival-time difference between any two transducers in all the experiments was $20 \mu$ s (run 4670) at the downstream boundary of the random medium and was $24 \mu \mathrm{s}$ (run 4708 ) after $22 \mathrm{~cm}$ of elear-air propagation. Assuming that the point with maximum slope on a nominally sinusoidally distorted wave front impacts the transducer, we obtain 5-6 $\mu$ s for the greatest possible rise time caused by this effect. Thus, titled shock fronts (more specifically, fronts distorted on the scale of $6 \mathrm{~cm}$ or so) could have caused at least some of the rather large effective rise times observed. As for the possibility that wrinkling of the shock front on a scale smaller than the diameter of the pressure transducer (say, on the scale of the microstructure $\gamma_{\rho}$ of 


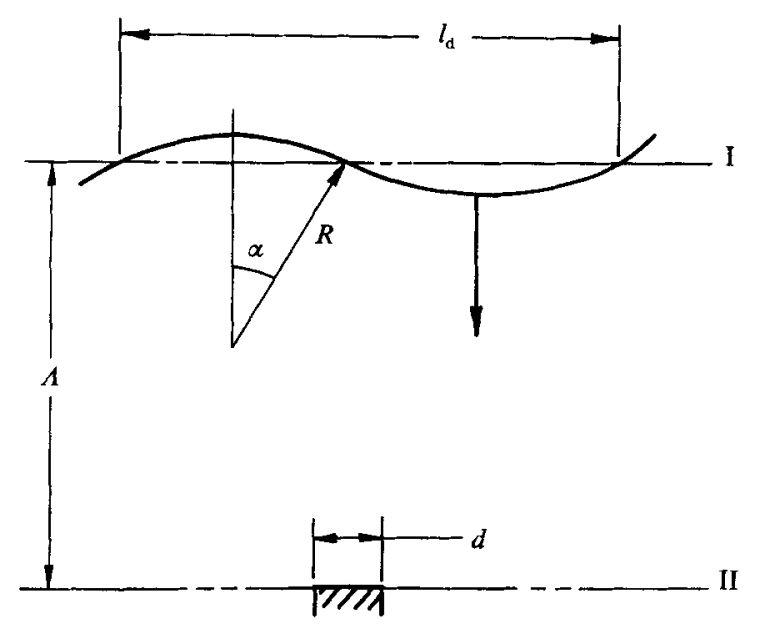

Figure 18. Schematic of a wrinkled wave front incident on a pressure transducer.

the density inhomogeneities) might cause large apparent rise times of the initial disturbance, wave-front distortions of up to $1.5 \mathrm{~mm}$ amplitude, with wavelengths of only $5 \mathrm{~mm}$, are simply not consistent with the photographic evidence of $\S 3.1$.

As already mentioned, it is significant that the distribution of measured rise times of the $M_{\mathrm{s}}=1.007$ shock waves after $22 \mathrm{~cm}$ of clear-air propagation is similar to those at the immediate downstream boundary of the scattering region. We first consider the wave-front geometry neglecting nonlinear steepening and then we discuss steepening effects. In figure 18 a shock wave moves from top to bottom, and pressure transducers are located at planes I and II separated by distance $A$. Distortions on the shock front at plane I have a lateral scale $l_{\mathrm{d}}$ and are of such an amplitude as to give a radius of curvature $R$. The size of the transducer $d$ is small compared with $l_{\mathrm{d}}$. We suppose that the slope of the wave $\alpha$ determines the apparent rise time of the transducer in plane I. According to geometric optics, after propagation of the shock to plane II, the magnitude of the slope $\alpha$ on the transducer in plane II must have decreased as long as $R<\frac{1}{2} \Lambda$, because the radius of curvature will have increased. Consequently, the apparent rise time due to tilt must decrease. On the other hand, if $R>\frac{1}{2} \Lambda$, in those few cases for which $R \sim A$ and also the wave is convergent onto plane II the rise time may increase to the effectively larger rise time of a single wrinkled front. Otherwise, it will also decrease. Only in the limiting case, when a substantial fraction of the sample of initial wave fronts has $R \approx \frac{1}{2} \Lambda$, so that focusing typically occurs about half-way between I and II, will the measured rise time at II be the same as at $I$. In the present experiments $A=22 \mathrm{~cm}$, so perturbations on the shock wave of $R \sim 10 \mathrm{~cm}$ with a lateral scale $l_{\mathrm{d}} \sim 6 \mathrm{~cm}$ would exhibit the same apparent rise time at $I$ as at II. We conclude from these observations that a substantial number of incident wave fronts in our experiments are distorted with $R \approx 10 \mathrm{~cm}$. It is interesting to compare this result with the radius of curvature that a shock would have if it originated as a spherically diverging wave at its first point of focus. The distance that an acoustic shock propagates through the medium of the present experiments before it first focuses with probability one can be calculated from the results of $\S 4.4$ to be about $5 \mathrm{~cm}$. Thus at the downstream station such a shock would have a radius of curvature of $30 \mathrm{~cm}$. The result that the actual value is 

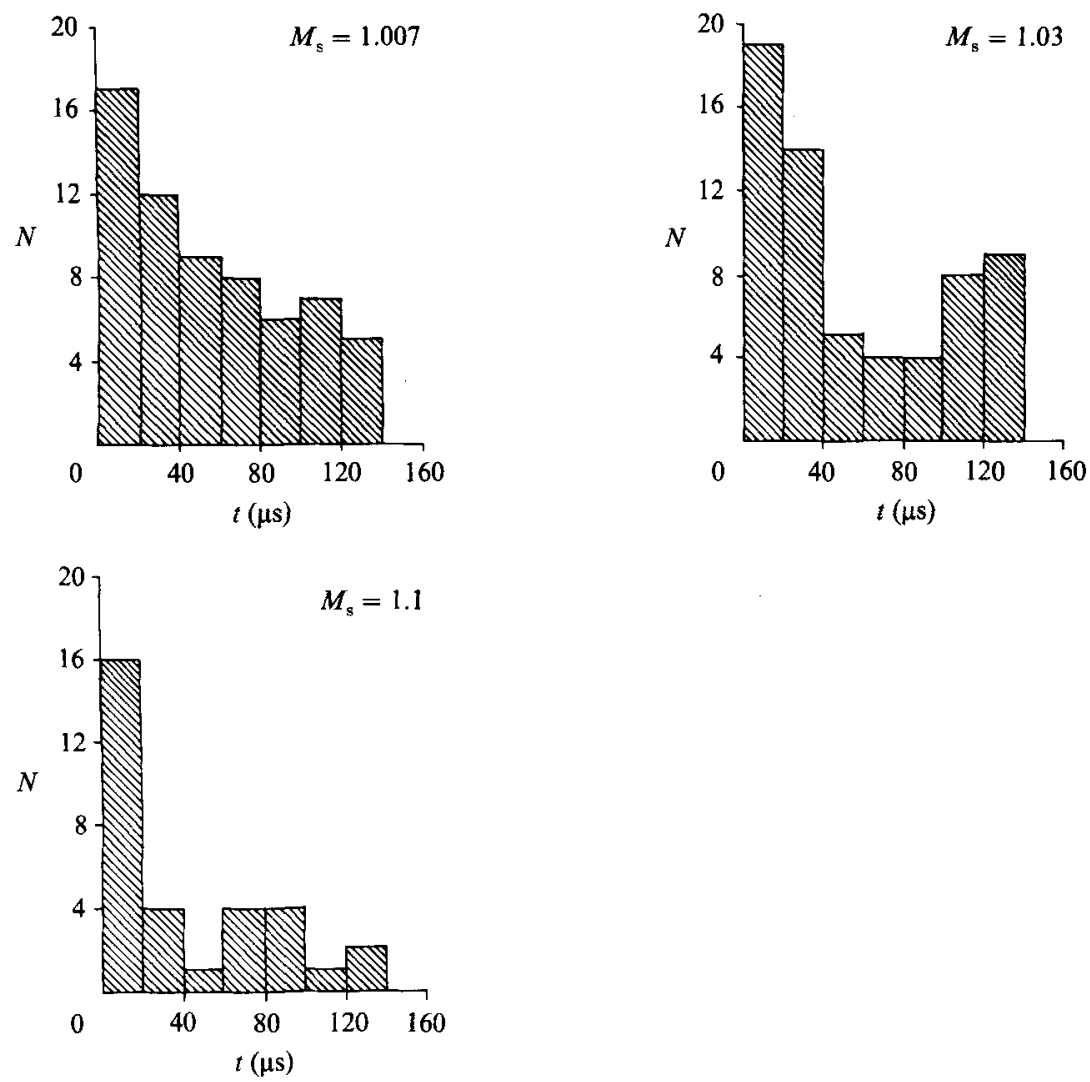

Figurf 19. Histograms for time to peak pressure measured at the exit-shutter station.

substantially smaller is consistent with the intuitive idea that the effective centre should be somewhere between the first focus and the measuring station.

\subsubsection{Time interval to peak pressure}

Histograms of the elapsed time between the main front and the maximum observed pressure are shown in figure 19. They show that the peak pressure occurs with highest probability within $20 \mu \mathrm{s}$ behind the front, but that a substantial number of samples of up to $120 \mu \mathrm{s}(40 \mathrm{~mm})$ occur. The mean values of the elapsed time between the shock front and the maximum pressure for the three cases $M_{\mathrm{s}}=1.007$, 1.03 and 1.1 are 58,52 and $36 \mu \mathrm{s}$, respectively.

Of the two points of view that can be adopted in considering the dispersion of shock waves in a random medium, we have adopted the one that emphasizes the geometrical nature of the process; namely, that the structure of the laboratory pressure traces and visualization photographs is attributable to a succession of scattered waves behind the initial disturbance. The second view, put forth by Plotkin \& George (1972) in a statistical theory utilizing a second-order perturbation scheme, gives the result that the scattering process disperses the shock wave in the same way that a greatly increased viscosity would. In fact, in their theory the effective viscosity turns out to be a sort of eddy viscosity which depends on the statistical properties of the medium. Thus, the thickness of, for example, weak shock waves is 

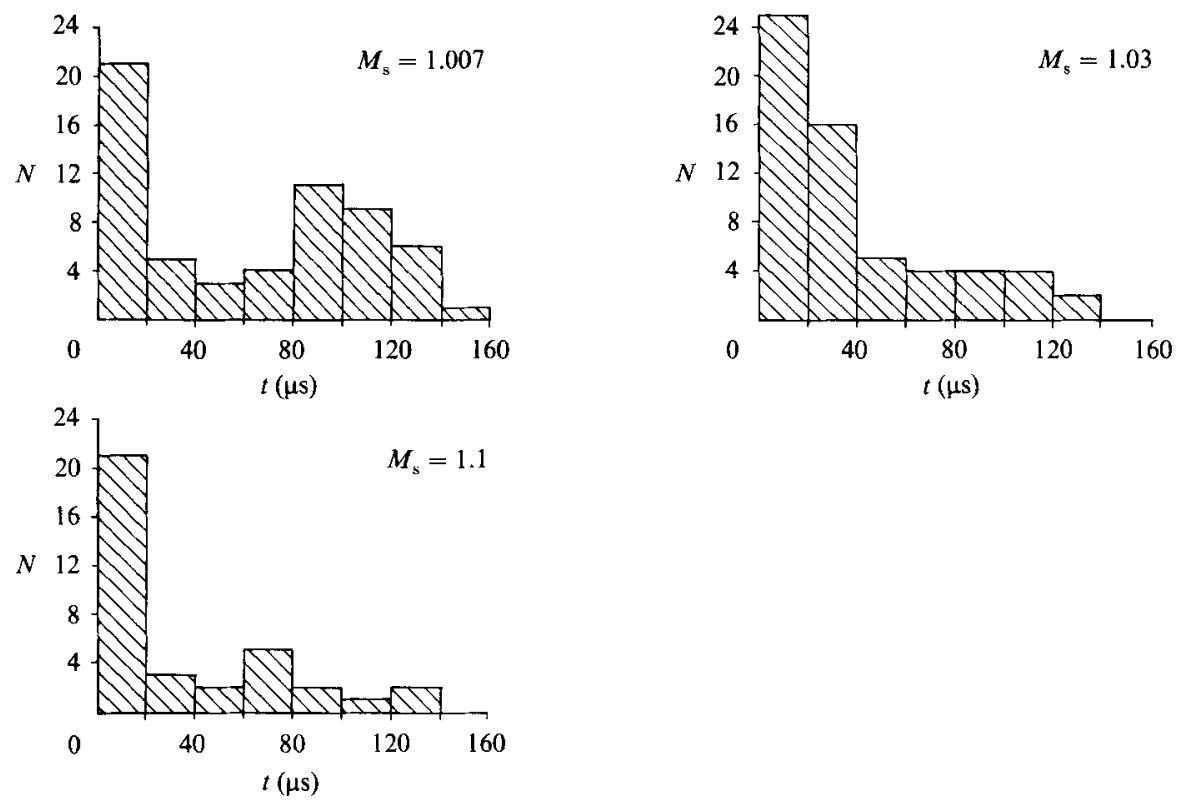

Figure 20. Histograms for time to peak pressure measured $21.6 \mathrm{~cm}$ beyond the exit-shutter station.

simply the Taylor thickness appropriate for a substance with this greatly increased viscosity.

The theory of Plotkin \& George describes a steady-state configuration in which the tendency of the random medium to disperse the wave is properly balanced by nonlinear steepening. An order-of-magnitude estimate, obtained by considering onedimensional non-steady characteristics, of the distance in which a wave of initial thickness $\delta$ and strength $M$ steepens to a discontinuity once it leaves the random medium is

$$
L \sim \frac{\delta}{2\left(M_{\mathrm{s}}-1\right)}
$$

Thus, shock waves of strength $M_{\mathrm{s}}=1.007,1.03$ and 1.1 with, say, rise times to peak pressure at plane $I$ (figure 18 ) less than about $8 \mu \mathrm{s}(\delta \leqslant 3 \mathrm{~mm}), 40 \mu \mathrm{s}(\delta \leqslant 10 \mathrm{~mm})$ and $125 \mu \mathrm{s}(\delta \leqslant 40 \mathrm{~mm})$, respectively, would steepen to discontinuities by the time they reached plane II $(L=22 \mathrm{~cm})$, but thicker waves would not. That is, by this argument the $M_{\mathrm{s}}=1.1$ and, perhaps, the $M_{\mathrm{s}}=1.03$ waves observed immediately downstream of the scattering medium should steepen to discontinuities in $22 \mathrm{~cm}$ of uniform propagation, while the $M_{\mathrm{s}}=1.007$ waves should not. In fact, as shown by comparing figure 20 with figure 19, the changes between stations I and II are rather small. This result suggests that the steepening argument cannot be applied in the region immediately downstream of strong scattering where significant focusing occurs.

\subsubsection{Shock distortion from arrival-time measurements}

The mean value of the differences between the time of arrival of the initial disturbance at the vertical and horizontal arrays in the measuring plane are tabulated in table 1 . The results indicate a slight vertical stratification; the 


\begin{tabular}{cccccc} 
& \multicolumn{2}{c}{ Shutter } & & \multicolumn{2}{c}{ Endwall } \\
\cline { 2 - 3 } \cline { 5 - 6 } \cline { 5 - 6 }$M$ & Horiz. & Vertical & & Horiz. & Vertical \\
1.007 & $\tau_{12}$ & $\tau_{34}$ & & $\tau_{12}$ & $\tau_{34}$ \\
1.03 & -2.46 & 14.92 & & -2.06 & 8.96 \\
1.1 & -0.82 & -7.31 & & 5.25 & 5.56 \\
$\tau_{i j}=$ & -3.0 & 2.38 & & -5.13 & 4.01 \\
difference between $t_{\mathrm{ab}}$ for transducers $i$ and $j$.
\end{tabular}

TABLE 1. Differences in arrival times

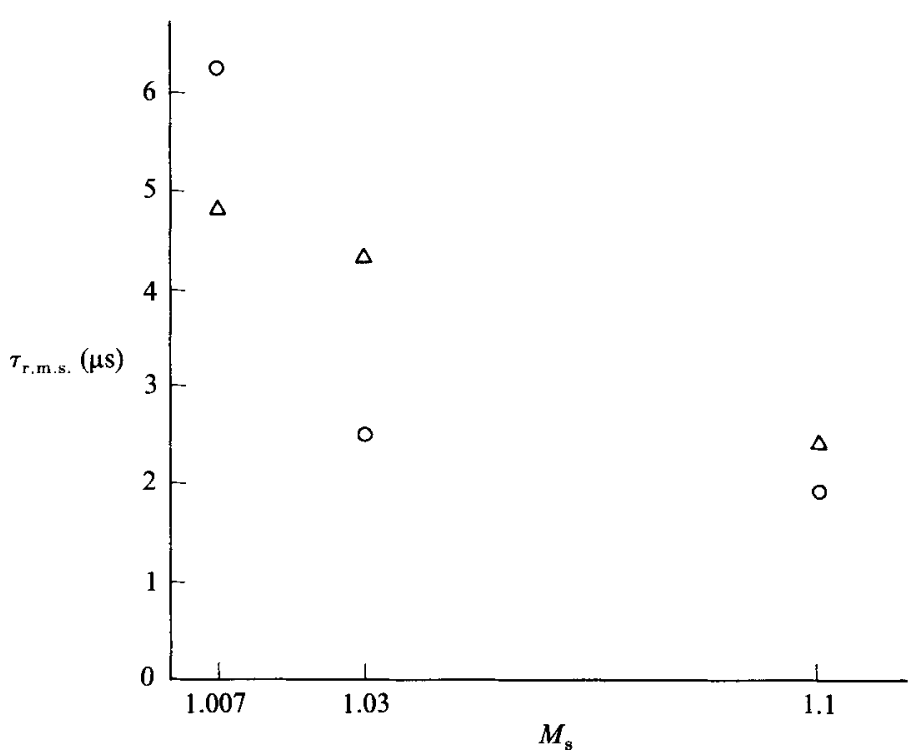

Figure 21. Arrival-time fluctuations of the initial disturbance, referenced to the arrival time of the same front at the centre transducer. Measurements are made at the exit-shutter location $(\triangle)$ and $21.6 \mathrm{em}$ beyond $(\mathrm{O})$.

maximum mean arrival-time difference between the top and bottom gauge is $14.9 \mu \mathrm{s}$, which corresponds to a $1.5 \%$ variation in the acoustic index of refraction.

The magnitude of the r.m.s. fluctuation of the arrival time of the initial disturbance at each transducer in the measuring plane, referenced to the arrival time of the same front at the centre transducer, is shown in figure 21. As expected, the stronger waves are considerably less sensitive to perturbation by the random medium. Also, modest reduction of the fluctuations, to nearly the resolution of the pressure transducers, is observed after $22 \mathrm{~cm}$ of clear-air propagation.

\subsubsection{Amplitude of the initial disturbance}

Histograms of the measured normalized shock pressure $p_{\text {cd }} / p_{\text {cd incident }}$ (cf. figure 16) are presented in figures 22 and 23 . The sample population is 64 for the $M_{\mathrm{s}}=1.007$ and $M_{\mathrm{s}}=1.03$ shocks and 32 for the $M_{\mathrm{s}}=1.1$ shocks. Measurements giving $p<1$ indicate attenuated shock waves while $p>1$ indicates waves stronger than the incident shock. The mean normalized shock strength is less than unity, as expected, except for the $M_{\mathrm{s}}=\mathbf{1 . 1}$ shock wave just downstream of the random medium. This 

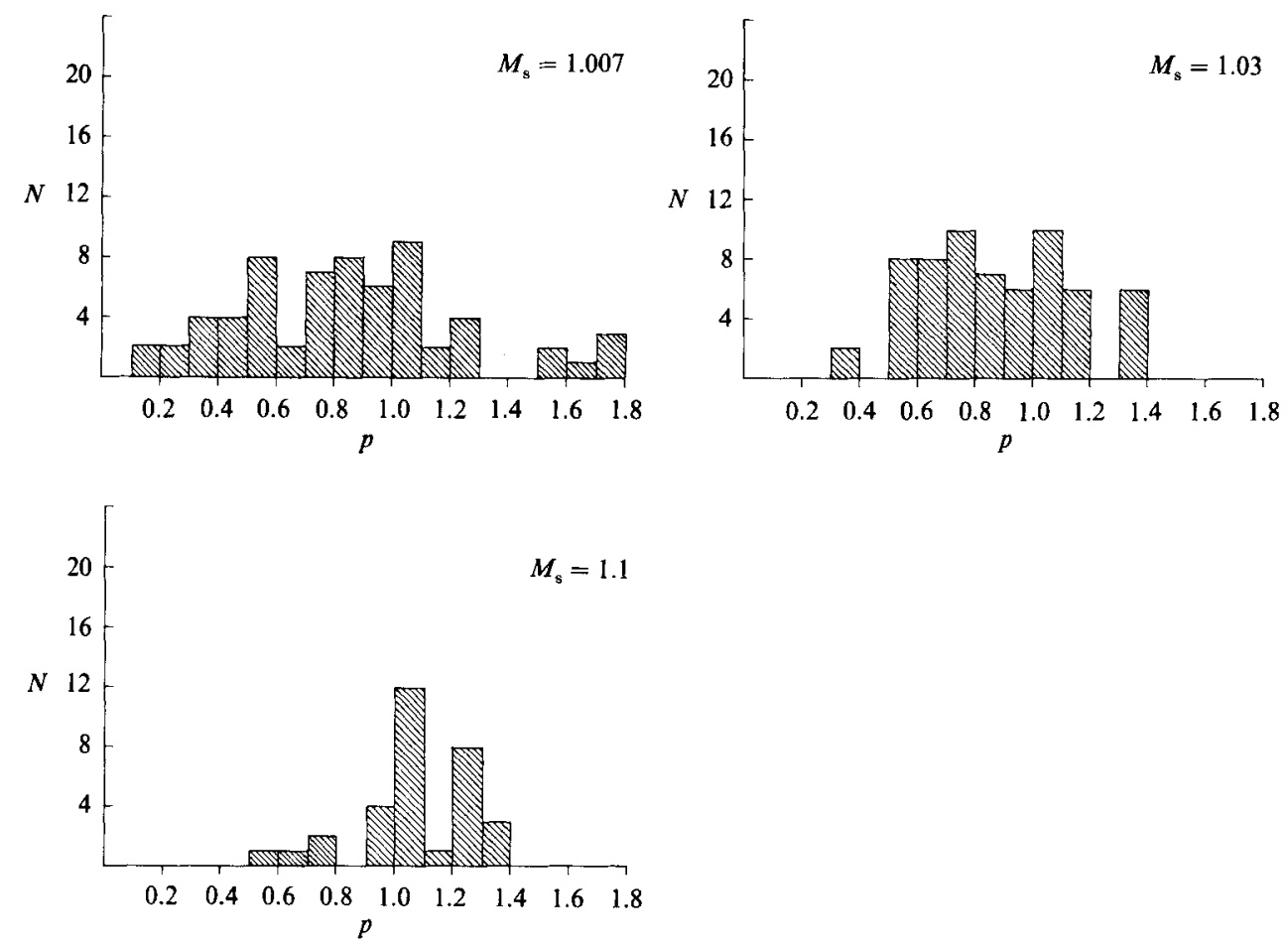

Figure 22. Histograms for shock pressure measured at the exit-shutter location.
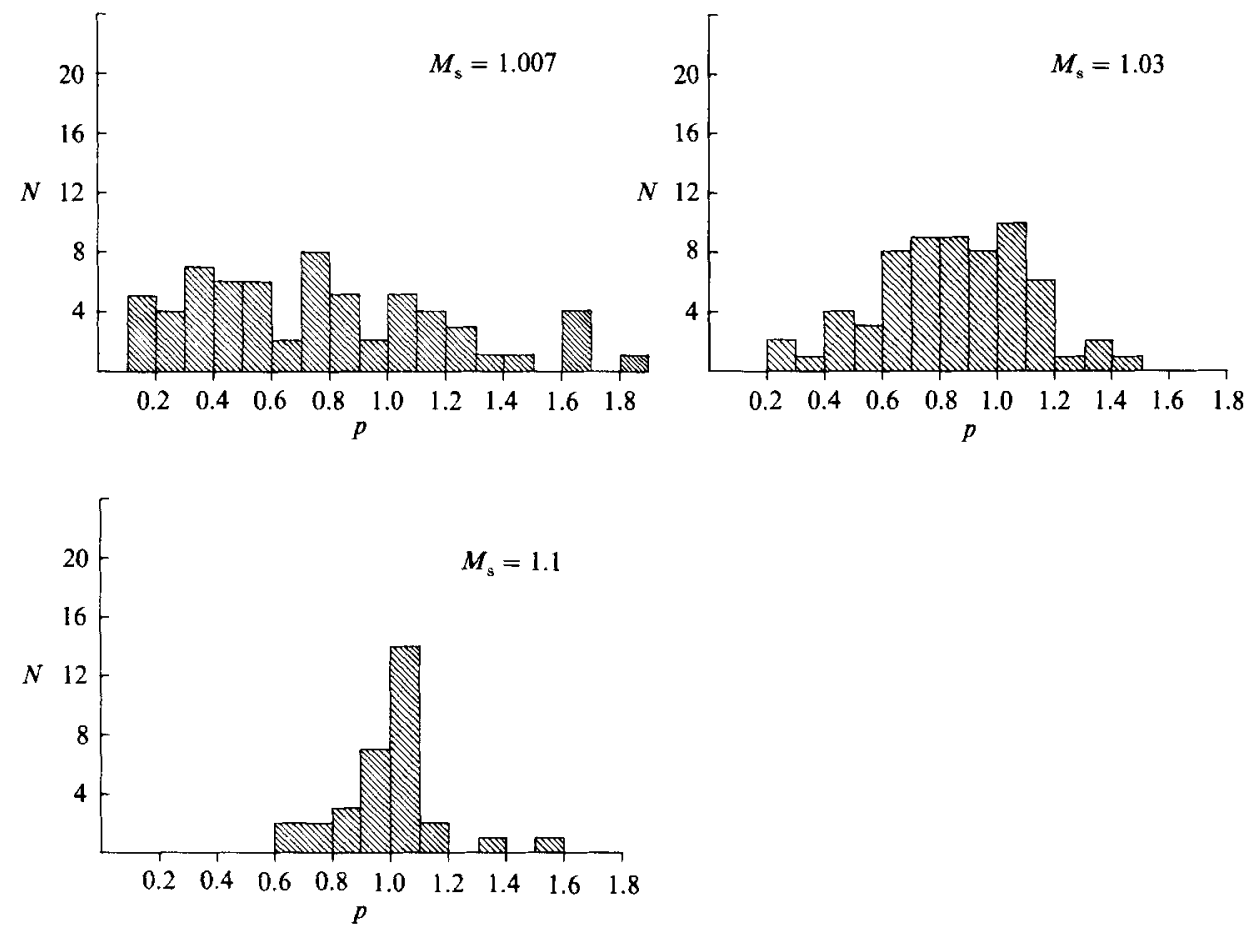

Figure 23. Histograms for shock pressure measured at the station $21.6 \mathrm{~cm}$ beyond the exit shutter. 


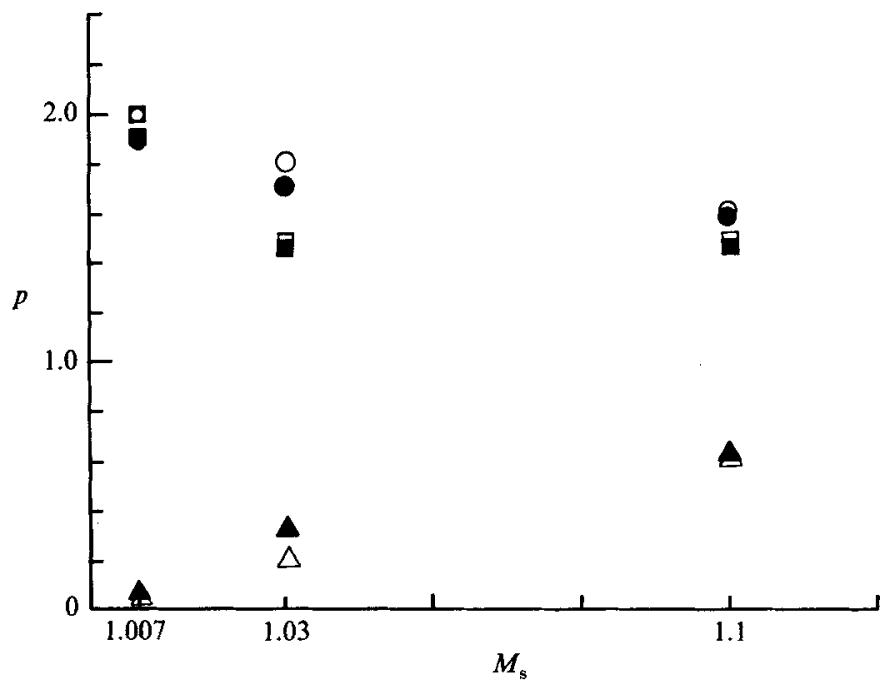

Figure 24. Maximum and minimum shock and peak pressures. The squares, circles and triangles denote the maximum normalized peak pressure and shock pressure and the minimum normalized shock pressure, respectively. The solid symbols represent measurements taken near the exit shutter and the open symbols denote measurements at a station $21.6 \mathrm{~cm}$ beyond.
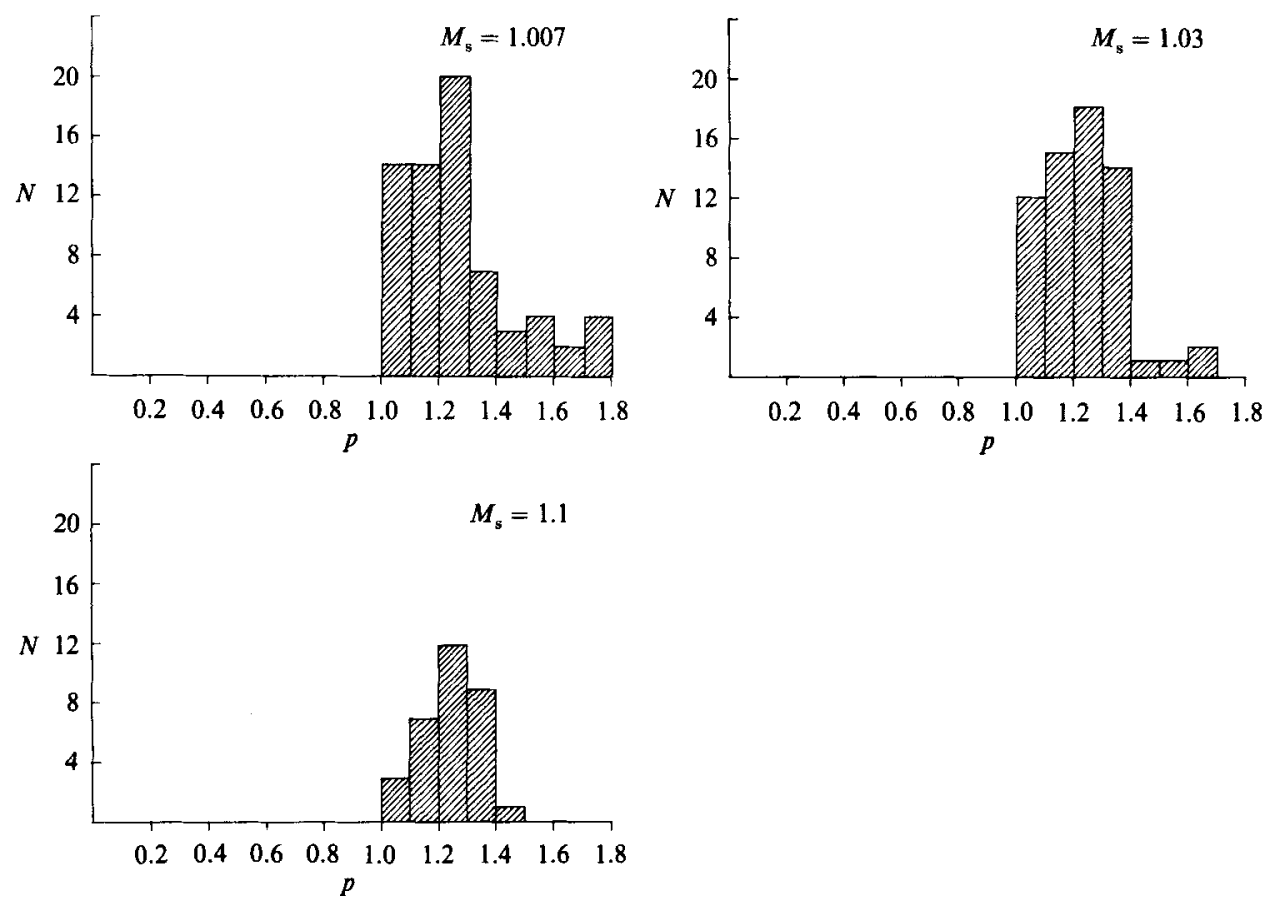

FIGURE 25. Histogram for the peak pressure measured at the exit-shutter location.

exception is probably due to the small sample population for that case. On the other hand, the mean (ensemble-averaged) pressure at much later times, after the major perturbations on the shock have passed the measuring station, is nearly equal to the unperturbed shock strength, so little energy is reflected upstream either at the boundaries of the random medium or from within it. 

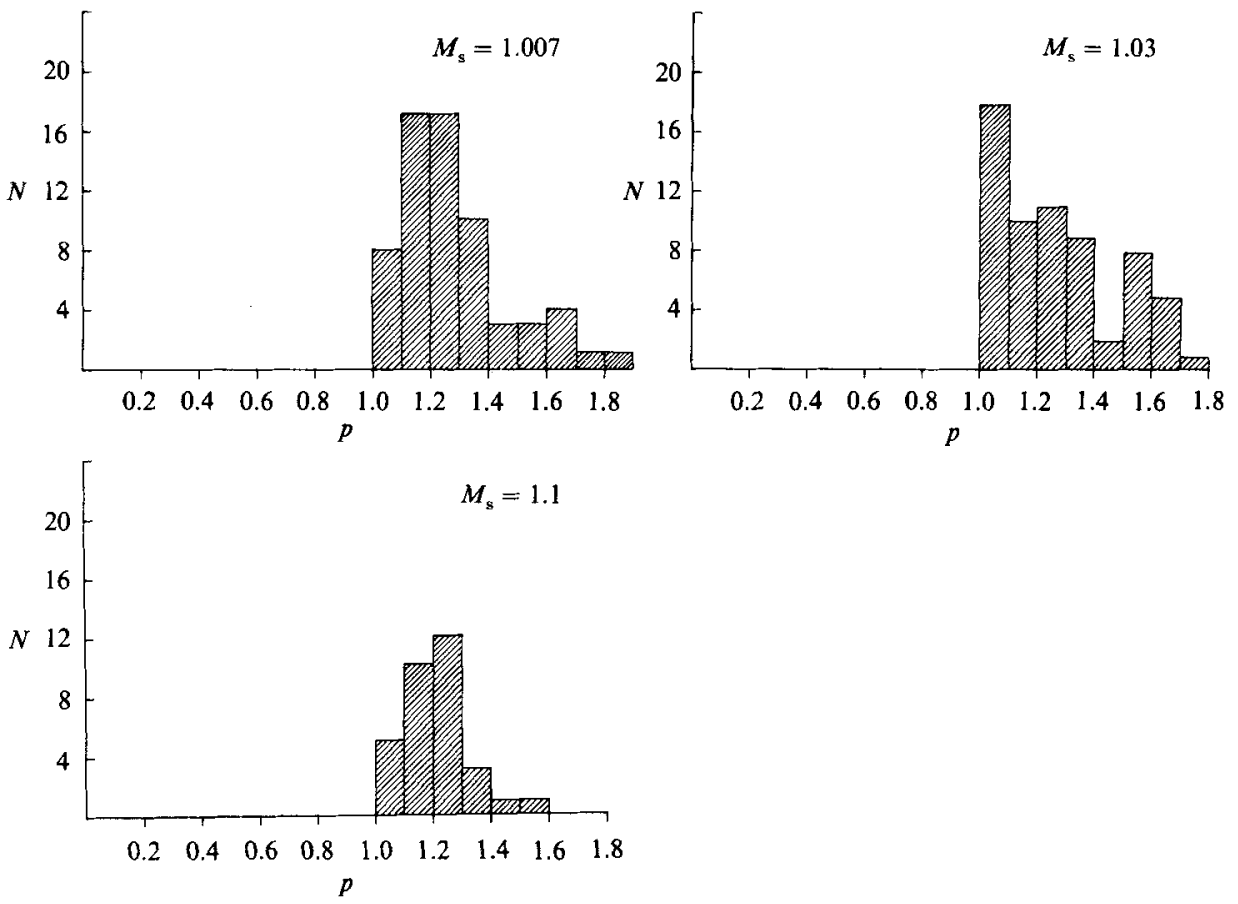

Figure 26. Histogram for the peak pressure measured at the station $21.6 \mathrm{~cm}$ beyond the exitshutter location.

Figure 24 shows the maximum and minimum observed values of shock amplitude at the two measuring stations. The minimum normalized shock amplitude increases and the maximum decreases with increasing Mach number, both trends being in agreement with the expected insensitivity of stronger shock waves to fluid inhomogeneity. This result can also be interpreted in terms of the behaviour of focusing shock waves, for it is known that the maximum amplification of a shock wave at a focus decreases with increasing shock strength owing to nonlinear effects which increase the size of the focal volume (Sturtevant \& Kulkarny 1976). It is interesting to note in figure 24 that the maximum observed shock amplitude is approximately 1.9 times the amplitude of the incident wave, nearly the same as observed in field studies of the focused sonic boom (Wanner et al. 1972) and in the laboratory by Sturtevant \& Kulkarny.

\subsubsection{Peak amplitude}

Figure 24 shows maximum observed values of peak amplitude (cf. figure 16). In figure 25 are presented histograms of the peak pressure at the station near the downstream boundary of the random medium, and in figure 26 are the results obtained $22 \mathrm{~cm}$ further downstream. It is evident that all peak pressures are larger than or equal to the strength of the shock incident upon the random medium. This is of course consistent with the fact that the average amplitude of the pressure signature far behind the front is, within the accuracy of the measurements, equal to the strength of the unmodified shock, and is at the same time, by definition, less than or equal to the maximum pressure. Figure 25 shows that near the downstream end of the random medium the most probable value of the peak pressure is 1.25 for all 
three Mach numbers. At the downstream station there is a tendency for the most probable peak pressure to be smaller.

\section{Interpretation of results by ray-tracing calculations}

The trajectories of the rays of acoustic waves through a computer-generated twodimensional random field which models the conditions of the present experiments have been calculated. The medium is computed by convolving a set of normally distributed random numbers (white noise) with a prescribed weighting function. The nonlinear ray equations are integrated using the method of characteristics. In the calculated scattering medium the fluctuation level of the acoustic index of refraction $(6 \%)$ and the average number of turbulent structures that the ray paths traverse (21.3) are the same as in the experiments. The computational mesh in the box is $128 \times 128$. By starting the calculations from any one of the four sides of the box, the same medium can be used as the source for four different propagation calculations.

\subsection{The computer-generated random medium}

The index-of-refraction field $n(x, y)$ can be represented by a convolution integral, in which a two-dimensional white-noise field $w(x, y)$ is smoothed by a weighting function $f(x, y)$ :

$$
n(x, y)=\iint_{-\infty}^{\infty} f\left(x-x^{\prime}, y-y^{\prime}\right) w\left(x^{\prime}, y^{\prime}\right) \mathrm{d} x^{\prime} \mathrm{d} y^{\prime}
$$

The purpose of the first part of the calculation is to determine $f(x, y)$. The twodimensional correlation function $R\left(x-x^{\prime}, y-y^{\prime}\right)$ of $n$ is

$$
\begin{aligned}
R\left(x-x^{\prime}, y-y^{\prime}\right) & =\left\langle n(x, y) n\left(x^{\prime}, y^{\prime}\right)\right\rangle \\
= & \left\langle\iiint \int_{-\infty}^{\infty} f\left(x-x^{\prime \prime}, y-y^{\prime \prime}\right) f\left(x^{\prime}-x^{\prime \prime \prime}, y^{\prime}-y^{\prime \prime \prime}\right) w\left(x^{\prime \prime}, y^{\prime \prime}\right) w\left(x^{\prime \prime \prime}, y^{\prime \prime \prime}\right)\right. \\
& \left.\times \mathrm{d} x^{\prime \prime} \mathrm{d} x^{\prime \prime \prime} \mathrm{d} y^{\prime \prime} \mathrm{d} y^{\prime \prime \prime}\right\rangle,
\end{aligned}
$$

where $\langle>$ denotes ensemble averaging. The white-noise field is uncorrelated, i.e.

$$
\left\langle w\left(x^{\prime \prime}, y^{\prime \prime}\right) w\left(x^{\prime \prime \prime}, y^{\prime \prime \prime}\right)\right\rangle=\delta\left(x^{\prime \prime}-x^{\prime \prime \prime}, y^{\prime \prime}-y^{\prime \prime \prime}\right),
$$

where $\delta(x, y)$ represents the Dirac delta function. Equations (4.2) and (4.3) may now be combined. After a change of variables,

$$
\alpha=x^{\prime \prime}-x, \quad \beta=y^{\prime \prime}-y,
$$

the result becomes (using symmetric weighting functions $f(x, y)$ )

$$
R\left(r_{1}, r_{2}\right)=\iint_{-\infty}^{\infty} f(\alpha, \beta) f\left(\alpha-r_{1}, \beta-r_{2}\right) \mathrm{d} \alpha \mathrm{d} \beta
$$

where

$$
r_{1}=\left|x^{\prime}-x\right|, \quad r_{2}=\left|y^{\prime}-y\right| \text {. }
$$

Equation (4.4) can easily be transformed into the frequency domain by taking a Fourier transform (FT) of both sides of the equation and expressing $\hat{f}\left(k_{1}, k_{2}\right)$, the $\mathbf{F T}$ of $f(x, y)$, in terms of $\hat{R}\left(k_{1}, k_{2}\right)$, the FT of $R\left(r_{1}, r_{2}\right)$

$$
\hat{f}\left(k_{1}, k_{2}\right)=\frac{1}{(2 \pi)^{\frac{1}{2}}} \hat{R}\left(k_{1}, k_{2}\right)
$$


In the absence of detailed information from the experiments, we assume that the correlation function is Gaussian with lengthscale $L_{\rho}$ (cf. §2.5.1). Thus,

$$
\begin{aligned}
\hat{R}\left(k_{1}, k_{2}\right) & =\mathrm{FT}\left\{\left\langle n^{2}\right\rangle \exp \left[-\frac{\left(r_{1}^{2}+r_{2}^{2}\right)}{2 L_{\rho}^{2}}\right]\right\} \\
& =\left\langle n^{2}\right\rangle L_{\rho}^{2} \exp \left\{-\frac{\left(k_{1}^{2}+k_{2}^{2}\right) L_{\rho}^{2}}{2}\right\} .
\end{aligned}
$$

Substituting (4.6) into (4.5) and taking the inverse FT of $\hat{f}\left(k_{1}, k_{2}\right)$ leads to the desired weighting function:

$$
f\left(r_{1}, r_{2}\right)=\left(\frac{2}{\pi}\right)^{\frac{1}{2}} \frac{1}{\mathrm{~L}_{\rho}}\left\langle n^{2}\right\rangle^{\frac{1}{2}} \exp \left\{-\frac{\left(r_{1}^{2}+r_{2}^{2}\right)}{L_{\rho}^{2}}\right\} .
$$

Now, from (4.7) and (4.1) we can obtain the index-of-refraction field,

$$
n(x, y)=\left(\frac{2}{\pi}\right)^{\frac{1}{2}} \frac{\left\langle n^{2}\right\rangle^{\frac{1}{2}}}{L_{\rho}} \iint_{-\infty}^{\infty} \exp \left\{-\frac{\left(x-x^{\prime}\right)^{2}+\left(y-y^{\prime}\right)^{2}}{L_{\rho}^{2}}\right\} w\left(x^{\prime}, y^{\prime}\right) \mathrm{d} x^{\prime} \mathrm{d} y^{\prime} .
$$

The ray-tracing calculations also require knowledge of the gradient of the indexof-refraction field in the $x$ - and $y$-directions. These quantities are obtained by differentiating (4.8):

$$
\begin{aligned}
& n_{x}(x, y)=-\frac{2^{\frac{3}{2}}}{\pi^{\frac{1}{2}}} \frac{\left\langle n^{2}\right\rangle^{\frac{1}{2}}}{L_{\rho}^{3}} \iint_{-\infty}^{\infty}\left(x-x^{\prime}\right) \exp \left\{-\frac{\left(x-x^{\prime}\right)^{2}+\left(y-y^{\prime}\right)^{2}}{L_{\rho}^{2}}\right\} w\left(x^{\prime}, y^{\prime}\right) \mathrm{d} x^{\prime} \mathrm{d} y^{\prime}, \\
& n_{y}(x, y)=-\frac{2^{\frac{3}{2}}}{\sqrt{ } \pi^{\frac{1}{2}}} \frac{\left\langle n^{2}\right\rangle^{\frac{1}{2}}}{L_{\rho}^{3}} \iint_{-\infty}^{\infty}\left(y-y^{\prime}\right) \exp \left\{-\frac{\left(x-x^{\prime}\right)^{2}+\left(y-y^{\prime}\right)^{2}}{L_{\rho}^{2}}\right\} \\
& \left.\times w\left(x^{\prime}\right), y^{\prime}\right) \mathrm{d} x^{\prime} \mathrm{d} y^{\prime} .
\end{aligned}
$$

Equations (4.8), (4.9) and (4.10) can be evaluated using fast Fourier transform (FFT) algorithms. The equations take the form of convolution integrals so the FT of $n(x, y)$ is proportional to the product of the Fourier-transformed Gaussian weighting function and the white-noise field:

$$
\mathrm{FT}\{n(x, y)\}=\left(\frac{2}{\pi}\right)^{\frac{1}{2}} \frac{\left\langle n^{2}\right\rangle^{\frac{1}{2}}}{L_{\rho}} 2 \pi \mathrm{FT}\left\{\exp \left[-\frac{x^{\prime 2}+y^{\prime 2}}{L_{\rho}^{2}}\right]\right\} \mathrm{FT}\left\{w\left(x^{\prime}, y^{\prime}\right)\right\}
$$

Taking the inverse FT of (4.11), we obtained the desired expression for the index-ofrefraction field:

$$
n(x, y)=(2 \pi)^{\frac{1}{2}} L_{\rho}\left\langle n^{2}\right\rangle^{\frac{1}{2}} \mathrm{FT}^{-1}\left\{\exp \left[-\frac{\left(k_{1}^{2}+k_{2}^{2}\right) L_{\rho}^{2}}{4}\right] \mathrm{FT}\left[w\left(x^{\prime}, y^{\prime}\right)\right]\right\} .
$$

Similarly, the expressions for $n_{x}(x, y)$ and $n_{y}(x, y)$ are

$$
\begin{aligned}
& n_{x}(x, y)=(2 \pi)^{\frac{1}{2}} L_{\rho}\left\langle n^{2}\right\rangle^{\frac{1}{2}} \mathrm{FT}^{-1}\left\{-\mathrm{i} k_{1} \exp \left[-\frac{4\left(k_{1}^{2}+k_{2}^{2}\right)}{L_{\rho}^{2}}\right] \mathrm{FT}\left[w\left(x^{\prime}, y^{\prime}\right)\right]\right\} \\
& n_{y}(x, y)=(2 \pi)^{\frac{1}{2}} L_{\rho}\left\langle n^{2}\right\rangle^{\frac{1}{2}} \mathrm{FT}^{-1}\left\{-\mathrm{i} k_{2} \exp \left[-\frac{4\left(k_{1}^{2}+k_{2}^{2}\right)}{L_{\rho}^{2}}\right] \mathrm{FT}\left[w\left(x^{\prime}, y^{\prime}\right)\right]\right\}
\end{aligned}
$$

\subsection{The ray-tracing procedure}

Numerical methods for the calculation of wave scattering have been reviewed by Clarebout (1976) and Candel $(1979 a, b)$, among others. Because in this study we are mainly interested in the geometry of the field, we have chosen to compute ray paths 
and wave fronts using the approach of geometrical optics, rather than a more complete diffraction method. The ray paths through the two-dimensional random medium are computed from the ray equations,

$$
\begin{gathered}
\frac{\mathrm{d}}{\mathrm{d} s} \nabla r=\nabla n, \\
\frac{\mathrm{d} \boldsymbol{x}}{\mathrm{d} s}=\frac{\nabla r}{n},
\end{gathered}
$$

where $s$ denotes the path length measured along the ray, $\boldsymbol{x}$ is the position vector of a point on the ray and $n$ is the index of refraction of the random medium. Initially, the rays are parallel to the $x$-axis. Subsequently, the ray position and the slope are computed for a small increment in path length $\Delta s$ using an iterative procedure. The index-of-refraction field is prescribed on a $128 \times 128$ mesh grid and intermediate values are computed by interpolation.

The wave fronts are surfaces of constant optical path length. Once the ray paths are known the path length along a ray can be computed as

$$
\int_{\text {ray }} n \mathrm{~d} s \text {. }
$$

In the numerical calculations the wave front is computed at five equally spaced locations in the direction of propagation.

\subsection{Results}

\subsubsection{Computer-generated medium and rays}

A photograph of the computer-generated medium is shown in figure 27 . The grey levels represent the value of the index of refraction; white indicates a high index and black a low index. The histogram at the bottom of the photograph is the histogram of grey levels, and thus gives the probability density function of the index-ofrefraction field. The results depicted in figure 29 are obtained by calculating rays through the medium of figure 27 incident on the left, top, right and bottom faces, respectively. As a check on the computer-generated medium, its radially symmetric correlation function is calculated numerically using Fourier-transform techniques (figure 28).

The ray paths and wave fronts of an initially plane wave which propagates through the computer-generated medium are shown in figure 29. The rays do not propagate along straight lines, but instead large excursions from the unperturbed path occur. It is instructive to consider the medium to be made up of a random array of gradient-index lenses which tend to focus and defocus portions of the wave front; the rays are deflected in the direction of the gradient of the index of refraction. Under the conditions set up to model the experiments, the first caustics form after only three or four correlation lengths. It is interesting to note that in most cases the bundle of rays that have been passed through a caustic can be identified as an expanding fan for at least ten correlation lengths beyond focus. Evidently, the distribution of the index-of-refraction field in the initial three to four correlation lengths of the medium strongly influences the behaviour of the rays downstream, at least over the propagation distance studied here. $\dagger$

+ Far inside the medium one experts the effects of the initial region to diminish, so the ray directions can be considered to be a Markovian process without after-effects (Kulkarny \& White 1982). 


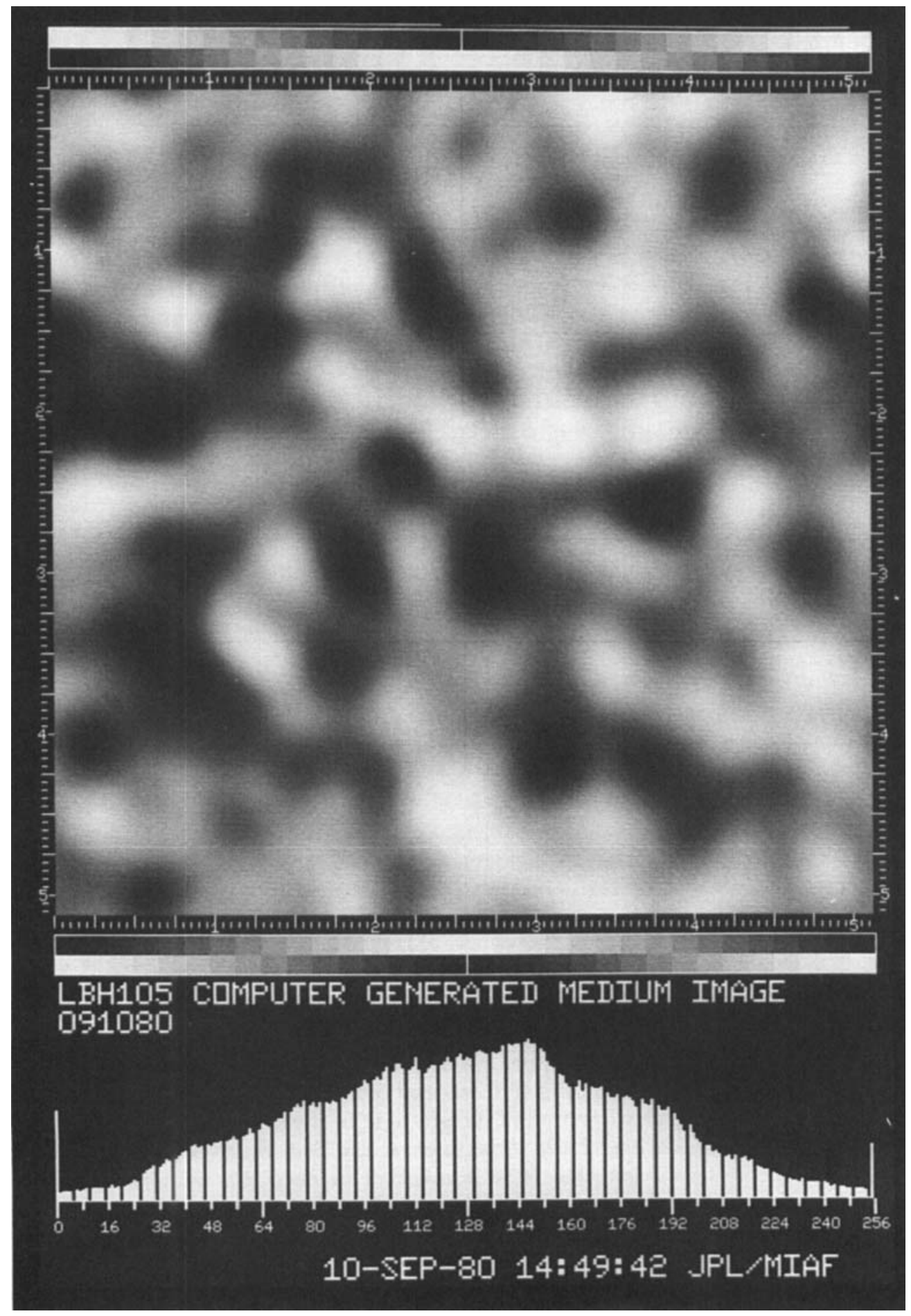

Figure 27. Grey-scale image of computer-generated medium. 


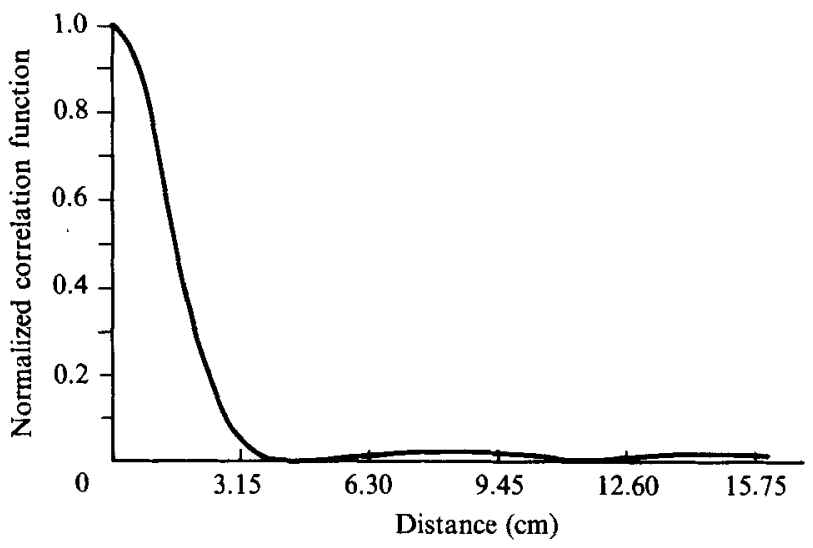

Figure 28. Radially symmetric correlation function of the computer-generated medium.

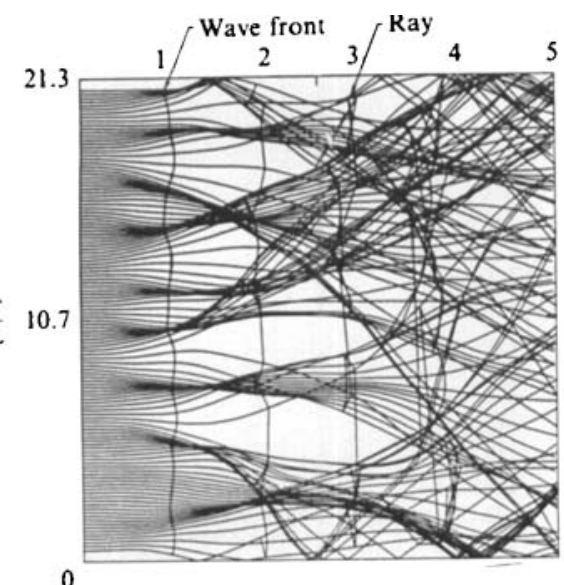

(a)

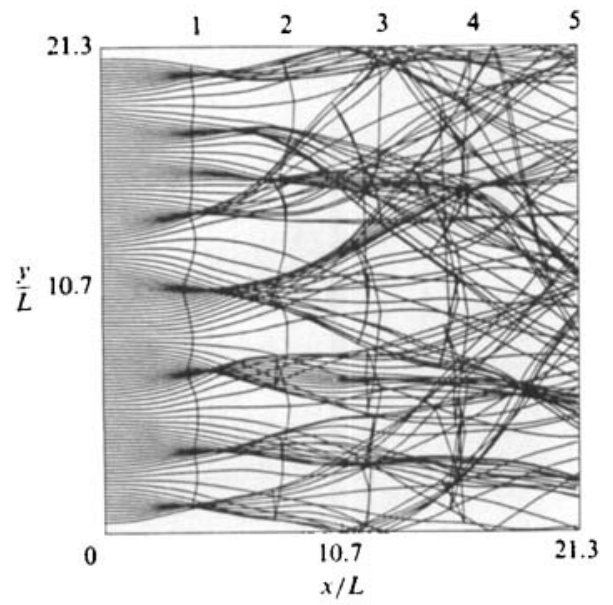

(c)

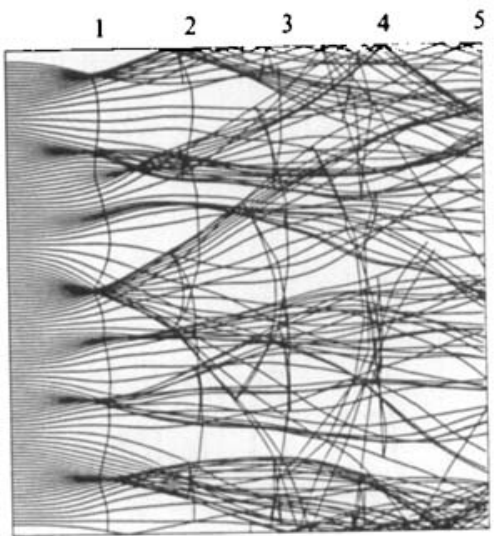

(b)

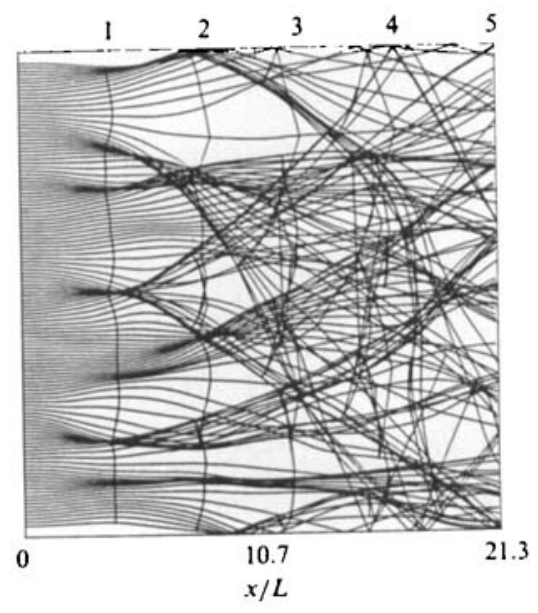

(d)

FigURE 29. Four representative ray-tracing results. (a) Corresponds to the unrotated medium as shown in figure 27 , and $(b),(c)$ and $(d)$ are obtained by counterclockwise rotation of the medium by $90^{\circ}, 180^{\circ}$ and $270^{\circ}$ respectively. 


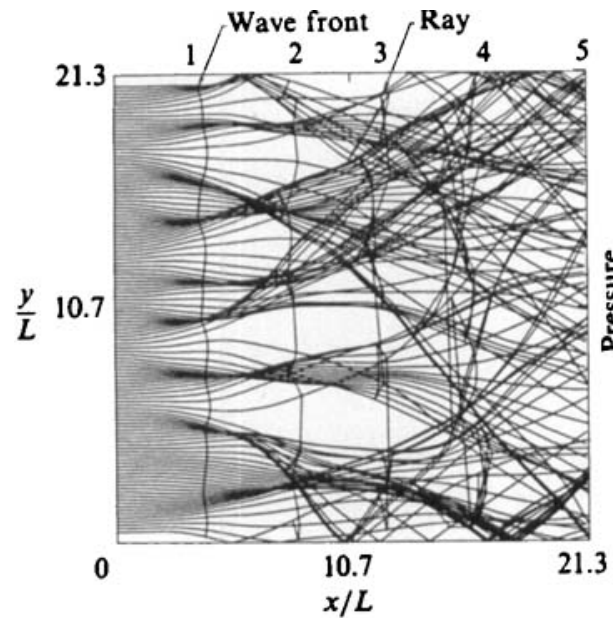

(a)

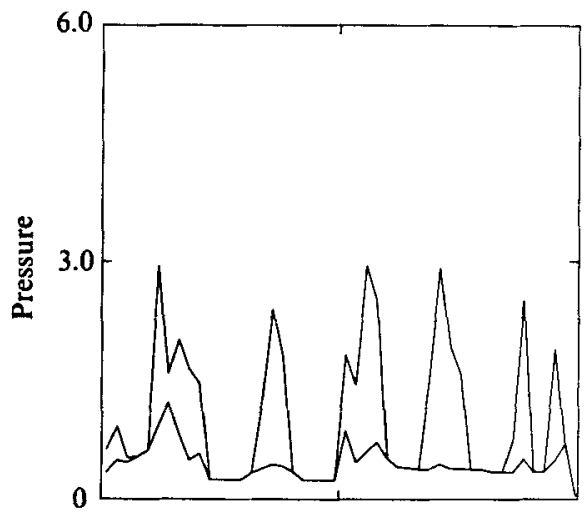

(c)

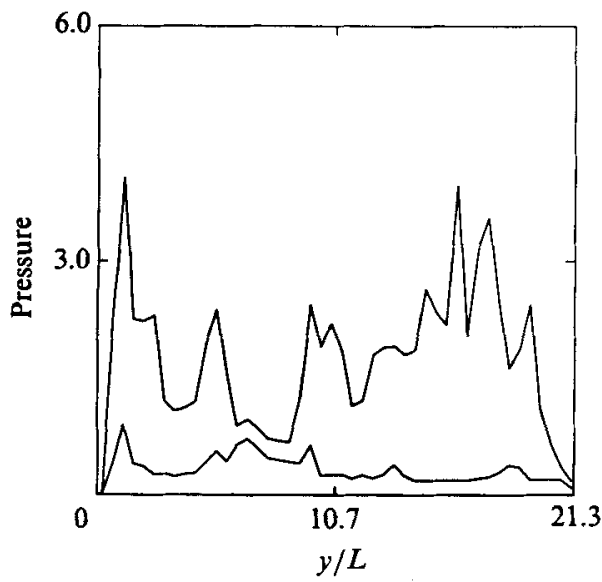

(e)

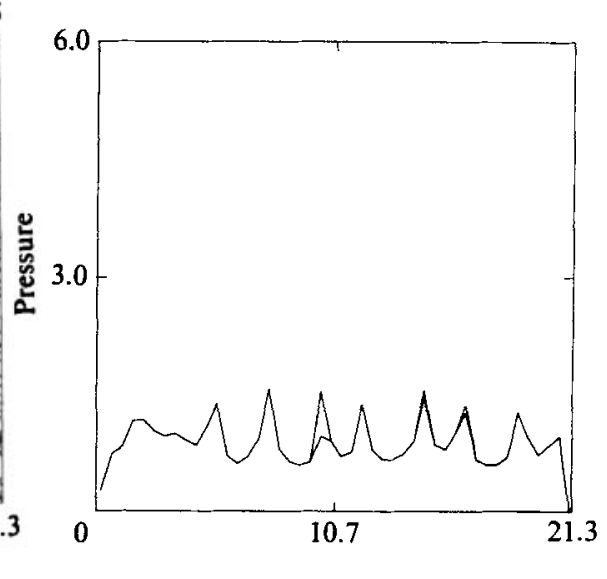

(b)

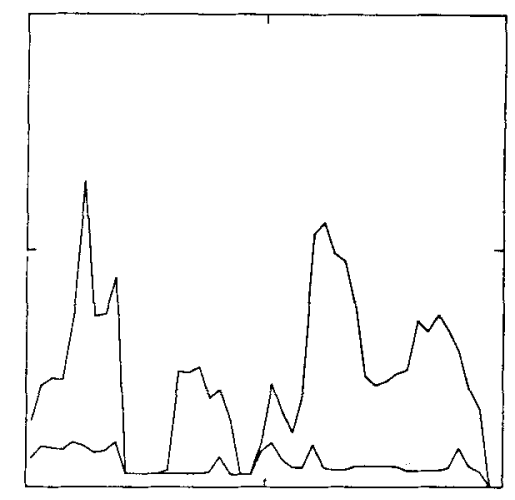

(d)

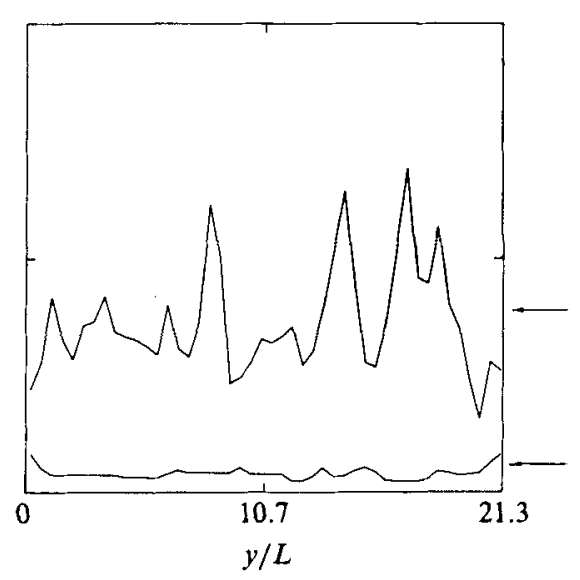

(f)

Figure 30. The evolution of wave amplitude and peak pressure as a function of propagation distance in the medium. (a) Shows the computed ray paths through the medium and in $(b)-(f)$ pressure profiles are shown; the top trace denotes peak pressure and the bottom trace wave-front pressure. Stations $(b)-(f)$ are located at $x / L=4,8,12,16$ and 20 , respectively. 


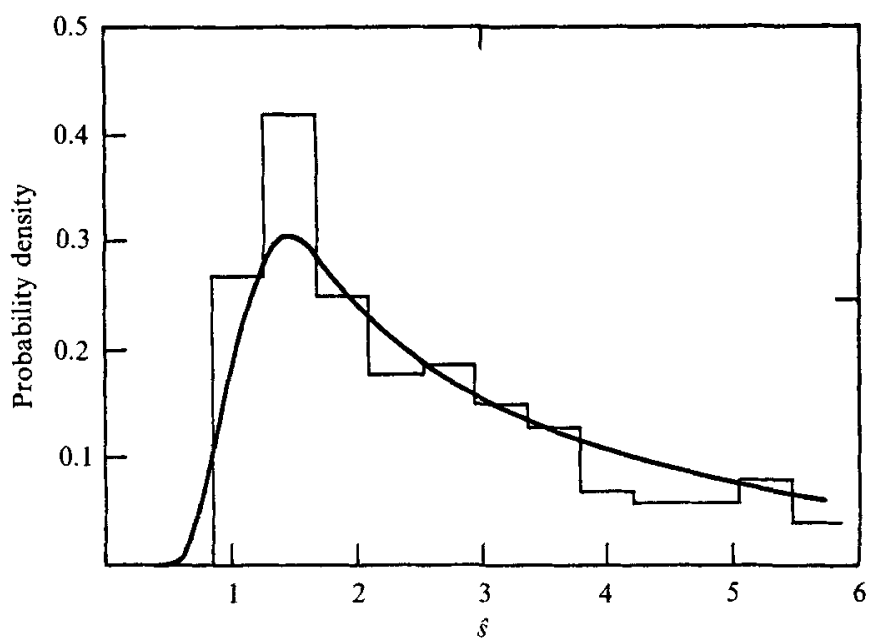

Figure 31. Comparison between the probability density function for the distance to first focus obtained from the numerical calculations and the Kulkarny-White analysis (curve).

The fact that whenever an acoustic wave passes through a focus the wave front becomes multiply folded (Friedlander 1958) is clearly demonstrated here. The pressure field is therefore made up of a succession of wave fronts. The area of the wave front and the wave-front thickness increase with propagation distance.

\subsubsection{Wavefront rise time}

In order to calculate the rise time of the initial disturbance, an imaginary row of pressure transducers is placed at each of the five stations at which the wave fronts are calculated. The transducer dimensions are chosen to correspond to the size of the transducers actually used in the experiments, so 46 transducers are distributed across the box of turbulence. A histogram of rise times is calculated using the data from all 46 transducers for the four orientations of the box and is compared to the experimental results in figure 17 (solid curve). The theoretical result for the most probable time is set equal to the experimental result, but otherwise no free parameters have been adjusted. The favourable comparison suggests that purely geometrical considerations are sufficient to explain the observed shock rise times.

The calculated p.d.f. is evidently nearly Gaussian in shape. Since the rays are perpendicular to the wave front, the ray directions are Gaussian distributed as well, in accord with Chernov's theory. However, that theory predicts a standard deviation that is twice as large as the one computed in our simulation. The discrepancy may be due to the large excursions the rays experience while travelling through the medium. Under such conditions the underlying assumptions of the Chernov theory are violated.

\subsubsection{Shock and peak amplitudes}

The amplitude of the initial wave front can be computed using the law of conservation of energy along a ray tube. In two dimensions the amplitude is inversely proportional to the square root of the distance between two points on adjacent rays. By this means we calculate the amplitude of that portion of the front that first strikes each transducer and average over all transducers to arrive at the shock amplitude. The peak amplitude attained behind the front is estimated by 
summing the contributions of all wave-front sections that strike the transducer. In figure 30 results are shown for the peak and wave-front amplitudes of the wave as it travels through the medium. At the first measuring station the peak amplitude occurs almost always at the shock. Thus the wave consists of a single front. Eventually the front distorts and becomes folded owing to focusing and the shock amplitude decreases owing to energy losses by scattering.

The maximum value of the peak pressure is limited by the transducer response; high pressures which occur over a small area are averaged over the transducer surface and this tends to reduce the observed peak values. Thus no very large amplitudes are observed, even in regions in which rays cross. The results obtained here cannot be directly compared with the experimental data, because of the neglect of diffraction effects. However, the simulation shows that locally high values of pressure can be observed, as well as some very low pressures at other locations. This observation is in agreement with measured flight test data and our shock-tube experiments.

\subsection{Distance to first focus}

The distance to first focus can be analysed by computing the distance to the first ray crossing between two adjacent rays. Following Kulkarny \& White (1982) a nondimensional distance to first focus is defined:

$$
\hat{s}=\sigma^{\frac{2}{3}} s,
$$

where

$$
\sigma^{2}=\int_{-\infty}^{\infty} \frac{\partial^{4} R}{\partial y^{4}}(x, 0) \mathrm{d} x
$$

and $R$ represents the correlation function of the medium. In our calculations the assumed functional form of $R$ is given by

$$
R(x, y)=\mu^{2} \exp \left(-\frac{x^{2}+y^{2}}{L_{\rho}^{2}}\right),
$$

where $\mu$ denotes the standard deviation of the index-of-refraction field, so

$$
\hat{s}=12^{\frac{1}{3}} \pi^{\frac{1}{6}} \mu^{\frac{2}{3}} \frac{s}{L_{\rho}} .
$$

According to Kulkarny \& White the p.d.f. of the distance to first focus measured along a ray peaks very sharply around $\hat{s}=1.3$, and then drops exponentially for large distances. Figure 31, constructed from our computed random medium, is in good agreement, the maximum occurring at $\hat{s} \approx 1.4$. The value of the most probable focal length depends only on the r.m.s. value of the index-of-refraction fluctuations and the form of the correlation function of the medium. No adjustable parameters are available. The dimensional form of this focal length, which we denote by $F_{p}$, can be directly computed from the Kulkarny-White analysis. The result for a medium characterized by a Gaussian correlation function is

$$
\begin{aligned}
F_{\mathrm{p}} & =1.4 \frac{\mathrm{L}_{\rho}}{\mu^{\frac{2}{3}} 12^{\frac{1}{3}} \pi^{\frac{1}{6}}} \\
& =0.51 \frac{L_{\rho}}{\mu^{\frac{2}{3}}} .
\end{aligned}
$$

Another quantity of interest, discussed in $\$ 3.2 .2$, is the mean radius of curvature of the shock fronts at a given image plane. Unfortunately, this quantity was not calculated by Kulkarny \& White. It should be noted that the calculations carried out 
here apply to the two-dimensional case. Recently Zwillinger \& White (1985) have extended the analysis to include three-dimensional effects. They found that even in that case rays tend to focus inside the medium at a preferred distance.

\section{Conclusions}

A unique laboratory apparatus has been constructed for the investigation of the interaction of weak shock waves with a statistically homogeneous random medium. The performance of the apparatus, which generates a mixture of helium and R12 with $6 \%$ r.m.s. sound-speed fluctuations, has been documented with point density measurements and baseline shock-wave tests, in which shock pressure histories with the medium fully-mixed and uniform were used to verify that the apparatus was properly balanced.

The flow before, during and after passage of the shock wave through the test section is visualized with schlieren and shadowgraph photography. A method for determining the power spectrum of the spatial scales of inhomogeneities in the random medium from a shadowgraph image has been applied to the field before passage of the shock. The effect of shock propagation on the properties of the random medium is most pronounced for strong waves, which, in turn, are little affected by the inhomogeneities. There is evidence of strong compression and enhanced mixing in the shock-processed fluid. Bright linear features on the schlieren photographs appear in the shocked fluid. They are preferentially oriented parallel to the shock front. Their separation indicates that the shocked fluid is substantially compressed. Similar trends are observed in the shadowgraph photos.

The images of distorted shock fronts on the photographs and the pressure traces are interpreted to represent a multiplicity of scattered wave fronts rather than a single highly wrinkled front. Quantitative measures of the scattering and distortion of the shock wave by the medium are obtained from the pressure measurements. Rounded and peaked pressure histories are observed, and the weaker waves show significant precursors. Statistics of shock pressures, peak pressures, arrival times, wave-front rise times, and the time interval to peak pressure are obtained from the pressure traces. The results are similar to those observed in flight tests (Maglieri \& Parrot 1963). Maximum overpressures about 1.9 times ambient are observed. Qualitatively, the occurrence of rounded and peaked pressure traces can be explained in terms of focusing and defocusing of the waves by the action of the random medium. Rise times two orders of magnitude larger than the Taylor thickness of unperturbed shock waves are observed. Rise-time measurements made in the region immediately downstream of the scattering medium suggest that the wave fronts emerging from the test region are randomly curved with radius of curvature of order $10 \mathrm{~cm}$ and lateral scale about $6 \mathrm{~cm}$, consistent with the photographic evidence. Comparison of the steepening time of a finite-amplitude wave with observations of the time interval to peak pressure after $22 \mathrm{~cm}$ of uniform propagation suggest that steepening arguments do not apply in regions where strong random focusing occurs, presumably because propagation paths are highly distorted and mixed.

Two-dimensional numerical ray-tracing calculations have been used to interpret the experimental observations. Diffraction effects, which are particularly important near a focus, have not been treated, but it is not expected that their inclusion would significantly alter our interpretations. It is found that rays tend to focus at a preferred distance from the entrance plane of the scattering medium. This distance, the most probable focal length $F_{p}$, is determined by the magnitude of the variations 
in the index of refraction of the inhomogeneities and by the derivatives of the correlation function. The calculations show that the trajectories are deterministic in appearance at least for the first few focal lengths. A simple scaling to conditions appropriate for sonic-boom propagation in the turbulent atmospheric boundary layer suggests that the propagation length through the layer is three or four most probable focal lengths, so the ray trajectories for the sonic boom should be similar to those computed in this study.

This work was supported under NSF Grant number MEA-8120092. L.H. acknowledges the support of the Dutch Government, a Fullbright Grant and a Josephine de Karman Fellowship. We are indebted to Professor H. W. Liepmann for stimulating support and Professor D. Coles who has made significant contributions to this work, in particular during the design stage of the experimental apparatus. The authors are also indebted to Dr B. White and Mr D. Madura and the Medical Image Analysis Facility, Jet Propulsion Laboratory, for their aid in processing data from photographs.

\section{REFERENCES}

Anyiwo, J. C. \& Bushneld, D. M. 1982 Turbulence amplification in shock-wave boundary-layer interaction. J. AIAA $20,893$.

Brown, G. L. \& Rebollo, M. R. 1972 A small, fast-response probe to measure composition in a binary gas mixture. AIAA J. 10, 649 .

CANDEL, S. M. 1979a Numerical solution of wave scattering problems in the parabolic approximation. J. Fluid Mech. 90, 465.

CANDEL, S. M. $1979 b$ Propagation of sound and ultra sound in non-homogeneous media. ONERA Rep. TP 1979-68.

Chu, B. T. \& Kovasznay, L. S. G. 1957 Non-linear interactions in a viscous heat-conducting compressible gas. J. Fluid Mech. 3, 494.

Clarebout, J. F. 1976 Fundamentals of Geophysical Data Processing. McGraw-Hill.

CRow, S. C. 1969 Distortion of sonic bangs by atmospheric turbulence. J. Fluid Mech. 37, 529.

Dettleff, G., Thompson, P. A., Meier, G. E. A. \& Speckmann, H.-D. 1979 An experimental study of liquefaction shock waves. $J$. Fluid Mech. 95, 279.

Ffowcs Wilutams, J. E. \& Howe, M. S. 1973 On the possibility of turbulent thickening of weak shock waves. J. Fluid Mech. 58, 461.

Friedlander, F. G. 1958 Sound Pulses. Cambridge University Press.

HaAs, J.-F. \& Sturtevant, B. 1987 Interaction of weak shock waves with cylindrical and spherical gas inhomogeneities. J. Fluid Mech. 181, 41.

Hesselink, L. 1976 Propagation of weak waves through a random medium. Bull. Am. Phys. Soc. $2,21,1226$.

Hesselink, L. 1977 An experimental investigation of propagation of weak shock waves in a random medium. Ph.D. thesis, California Institute of Technology, Pasadena, CA.

Hesselink, L. \& White, B. S. 1983 Digital image processing of flow visualization photographs. Appl. Opt. 22, 1454.

Hodason, J. P. 1973 Vibrational relaxation effects in weak shock waves in air and the structure of sonic bangs. J. Fluid Mech. 58, 187.

HowE, M.S. 1973 Corrigendum: Multiple seattering of sound by turbulence and other inhomogeneities. J. Sound Vib. 29, 398.

Keller, J. B. 1962 Hydrodynamic Instability, p. 227. American Mathematical Society, Providence.

Kravtsov, YU. A. 1969 The geometric optics approximation in the general case of inhomogeneous and nonstationary media with frequency and spatial dispersion. Sov. Phys. JETP 28, 769. 
Kulkarny, V. A. \& White, B. 1982 Focusing of waves in turbulent inhomogeneous media. Phys. Fluids 25, 1770.

Lighthill, M. J. 1953 Interaction of turbulence with sound or shock waves. Proc. Camb. Phil. Soc. 49, 531 .

Maglieri, D. J. \& Parrot, T. L. 1963 Lateral spread sonic-boom ground-pressure measurements from airplanes at altitudes to $75000 \mathrm{ft}$ and at Mach numbers to 2. NASA Tech. Note D2021.

Markstein, G. H. 1957 a Flow disturbances induced near a slightly wavy contact surface, or flame front, traversed by a shock wave. J. Aero. Sci. 24, 238.

Markstein, G. H. $1957 b$ A shock tube study of flame front-pressure wave interactions. 6th Intl Symp. Combustion, p. 387. Reinhold.

Meshkov, Y. Y. 1970 Instability of a shock wave accelerated interface between two gases. NASA $T T F-13,074$.

MEYER, K. A. \& BLEWETt, P.J. 1972 Numerical investigation of the stability of a shock accelerated interface between two fluids. Phys. Fluids 15, 753 .

Picone, J. M., Oran, E. S., Boris, J. P.\& Young, T. R. JR. 1984 Theory of vorticity generation by shock wave and flame interactions. NRL Memo. Rep. 5366.

Pierce, A. D. 1968 Spikes on sonic-boom pressure waveforms. J. Acoust. Soc. Am. 44, 1052.

Plotkin, K. J. \& George, A. R. 1972 Propagation of weak shock waves through turbulence. $J$. Fluid Mech. 54, 449.

Ribner, H. S. 1954 Shock-turbulence interaction and the generation of noise. NACA Tech. Note 3255 .

Richtmy eR, R. D. 1960 Taylor instability in shock acceleration of compressible fluids. Commun. Pure Appl. Maths 23, 297.

Rudinger, G. 1958 Shock wave and flame interactions. In Combustion and Propulsion, pp. 153-82. Third AGARD Coll. London. Pergamon.

Rudinger, G. \& Somers, L. 1960 Behaviour of small regions of different gases carried in accelerated gas flows. J. Fluid Mech. 7, 161.

Sturtevant, B. \& Kulkarny, V. A. 1976 The focusing of weak shock waves. J. Fluid Mech. 73, 651.

TAYLOR, G. I. 1950 The instability of liquid surfaces when accelerated in a direction perpendicular to their plane. I. Proc. R. Soc. Lond. A 201, 192.

Townsend, A. A. 1976 The Structure of Turbulent Shear Flow, Chap. 3. Cambridge University Press.

Wanner, J. C., Valee, J., Vivier, C. \& Thiery, C. 1972 Theoretical and experimental studies of the focus of sonic booms. J. Acoust. Soc. Am. 52, 13.

Werner, J. E. 1961 Unsteady interaction of a shock wave with a cellular vortex field. $J$. Fluid Mech. 10, 195.

White, B. S. 1987 Random rays and stochastic caustics. In Random Media (ed. G. Papanicolaou), The IMA Volumes in Mathematics and its Application, vol. 7, p. 311. Springer.

Whitham, G. B. 1974 Linear and Nonlinear Waves. Wiley.

Winkler, K.-H. A., Chalmers, J. W., Hodson, S. W., Woodward, P. R. \& Zabusky, N. J. 1987 A numerical laboratory. Physics Today, October, p. 28.

Zwillinger, D. I. \& White, B.S. 1985 Propagation of initially plane waves in the region of random caustics. Wave Motion 7, 207. 